\title{
Sharp spectral stability estimates via the Lebesgue measure of domains for higher order elliptic operators*
}

\author{
Victor I. Burenkov and Pier Domenico Lamberti ${ }^{\dagger}$
}

November 11, 2018

\begin{abstract}
We prove sharp stability estimates for the variation of the eigenvalues of non-negative self-adjoint elliptic operators of arbitrary even order upon variation of the open sets on which they are defined. These estimates are expressed in terms of the Lebesgue measure of the symmetric difference of the open sets. Both Dirichlet and Neumann boundary conditions are considered.
\end{abstract}

Keywords: Elliptic equations, Dirichlet and Neumann boundary conditions, stability of eigenvalues, sharp estimates, domain perturbation.

2000 Mathematics Subject Classification: 35P15, 35J40, 47A75, 47B25.

\section{Introduction}

We consider a non-negative self-adjoint operator

$$
H u=(-1)^{m} \sum_{|\alpha|=|\beta|=m} D^{\alpha}\left(A_{\alpha \beta}(x) D^{\beta} u\right), \quad x \in \Omega,
$$

of order $2 m$ subject to homogeneous Dirichlet or Neumann boundary conditions on a bounded open set $\Omega$ in $\mathbb{R}^{N}$. Here $m \in \mathbb{N}$ is arbitrary and the coefficients $A_{\alpha \beta}$ are bounded measurable functions satisfying the uniform ellipticity condition.

If $\Omega$ is sufficiently regular then $H$ has compact resolvent and its spectrum consists of a sequence of eigenvalues

$$
\lambda_{1}[\Omega] \leq \lambda_{2}[\Omega] \leq \cdots \leq \lambda_{n}[\Omega] \leq \cdots
$$

of finite multiplicity such that $\lim _{n \rightarrow \infty} \lambda_{n}[\Omega]=\infty$.

In this paper, for fixed coefficients $A_{\alpha \beta}$, we prove sharp stability estimates for the variation of $\lambda_{n}[\Omega]$ upon variation of $\Omega$.

The problem of estimating the deviation of the eigenvalues of second order elliptic operators following a domain perturbation has been considered by several authors: we refer to Burenkov, Lamberti and Lanza de Cristoforis [9] for extensive references on this subject and to Barbatis, Burenkov and Lamberti [3] for a recent

\footnotetext{
*to appear in Revista Matemática Complutense, DOI 10.1007/s13163-011-0079-2

${ }^{\dagger}$ Corresponding Author
} 
paper concerning stability estimates for resolvents, eigenfunctions and eigenvalues in the case of domain perturbations obtained by suitable diffeomorphisms.

The case of higher order operators has been far less investigated. We refer to Prikazhchikov and Klunnik [11] for the case of the biharmonic operator subject to Dirichlet boundary conditions on smooth open sets and to Burenkov and Lamberti [6] for the general case of higher order elliptic operators subject to Dirichlet or Neumann boundary conditions on open sets with continuous boundaries. The estimates provided in [6],[11] are expressed in terms of the Hausdorff distance between the open sets.

In this paper we develop the approach of Burenkov and Lamberti [7, 8] aiming at estimates via the Lebesgue measure of the symmetric difference of the open sets.

Namely, we consider families of open sets which are locally subgraphs of functions of class $C^{m-1,1}$. We require that the 'atlas' $\mathcal{A}$, with the help of which such boundaries are described, is fixed and we consider the class $C_{M}^{m-1,1}(\mathcal{A})$ of open sets for which the behavior of the derivatives of the functions describing the boundaries is controlled by a fixed constant $M>0$ (see Definition 2.10).

Let $\varphi_{n}[\Omega], n \in \mathbb{N}$, denote an orthonormal sequence of eigenfunctions corresponding to the eigenvalues $\lambda_{n}[\Omega]$. In Corollary 4.14 we prove that if $\mathfrak{A}$ is a family of open sets of class $C_{M}^{m-1,1}(\mathcal{A})$ such that for some $2<p \leq \infty$

$$
\sup _{\Omega \in \mathfrak{A}}\left\|\varphi_{n}[\Omega]\right\|_{W^{m, p}(\Omega)}<\infty
$$

for all $n \in \mathbb{N}$, then for each $n \in \mathbb{N}$ there exists $c_{n}>0$ such that for both Dirichlet and Neumann boundary conditions

$$
\left|\lambda_{n}\left[\Omega_{1}\right]-\lambda_{n}\left[\Omega_{2}\right]\right| \leq c_{n}\left|\Omega_{1} \Delta \Omega_{2}\right|^{1-\frac{2}{p}}
$$

for all $\Omega_{1}, \Omega_{2} \in \mathfrak{A}$ satisfying $\left|\Omega_{1} \Delta \Omega_{2}\right|<c_{n}^{-1}$, where $\left|\Omega_{1} \Delta \Omega_{2}\right|$ is the Lebesgue measure of the symmetric difference $\Omega_{1} \triangle \Omega_{2}$.

If $\Omega_{1}$ is fixed and $\Omega_{2} \subset \Omega_{1}$ then in the case of Dirichlet boundary conditions the assumptions of Corollary 4.14 can be weakened. In fact, in Corollary 4.12 we prove that if $\Omega_{1}$ is of class $C_{M}^{m-1,1}(\mathcal{A})$ and, for some $2<p \leq \infty, \varphi_{n}\left[\Omega_{1}\right] \in W^{m, p}\left(\Omega_{1}\right)$ for all $n \in \mathbb{N}$, then for each $n \in \mathbb{N}$ there exists $c_{n}>0$ such that for Dirichlet boundary conditions

$$
\lambda_{n}\left[\Omega_{1}\right] \leq \lambda_{n}\left[\Omega_{2}\right] \leq \lambda_{n}\left[\Omega_{1}\right]+c_{n}\left|\Omega_{1} \backslash \Omega_{2}\right|^{1-\frac{2}{p}}
$$

for all $\Omega_{2}$ of class $C_{M}^{m-1,1}(\mathcal{A})$ satisfying $\Omega_{2} \subset \Omega_{1}$ and $\left|\Omega_{1} \backslash \Omega_{2}\right| \leq c_{n}^{-1}$. (In this case there are no assumptions on the eigenfunctions $\varphi_{n}\left[\Omega_{2}\right]$.)

In Section 5 we also prove that, in general, the exponent $1-2 / p$ in (1.3) and (1.4) cannot be replaced by a larger one.

If the coefficients $A_{\alpha \beta}$ are of class $C^{m}$ and the open sets are of class $C^{2 m}$, condition (1.2) is satisfied with $p=\infty$. It follows that for each $n \in \mathbb{N}$ there exists $c_{n}>0$ such that

$$
\left|\lambda_{n}\left[\Omega_{1}\right]-\lambda_{n}\left[\Omega_{2}\right]\right| \leq c_{n}\left|\Omega_{1} \Delta \Omega_{2}\right|,
$$

for all $\Omega_{1}, \Omega_{2}$ of class $C_{M}^{2 m}(\mathcal{A})$ satisfying $\left|\Omega_{1} \Delta \Omega_{2}\right|<c_{n}^{-1}$. See Corollary 4.20.

The case $m=1$ was considered in [7, 8]. As in [7, 8], the proof of our estimates is based on the general spectral stability theorem [8, Thm. 3.2]. In order to 
apply that theorem we construct linear operators $\mathcal{T}_{\mathcal{D}}: W_{0}^{m, 2}\left(\Omega_{1}\right) \rightarrow W_{0}^{m, 2}\left(\Omega_{2}\right)$, $\mathcal{T}_{\mathcal{N}}: W^{m, 2}\left(\Omega_{1}\right) \rightarrow W^{m, 2}\left(\Omega_{2}\right)$ possessing a number of special properties. These operators serve as 'transition operators' for Dirichlet and Neumann boundary conditions respectively, as required by the general spectral stability theorem. We point out that the construction of such transition operators for $m>1$ is rather sofisticated and a straightforward extension to the case $m>1$ of the techniques used in [7] for $m=1$ is not possible (see the beginning of Section 3 for details).

We note that in [6] we proved spectral stability estimates expressed in terms of so-called 'atlas' distance introduced in [6, Definition 5.1] and of the Hausdorff distance of the boundaries of $\Omega_{1}$ and $\Omega_{2}$. In that case we considered classes of open sets with boundaries admitting arbitrarily strong degenerations and we did not require any summability assumption on the eigenfunctions and their gradients. However, as we pointed out in [7, Example 8.1], using the Lebesgue measure of $\Omega_{1} \triangle \Omega_{2}$ as we do here, allows to obtain better estimates.

\section{Preliminaries and notation}

Let $N, m \in \mathbb{N}$ and $\Omega$ be an open set in $\mathbb{R}^{N}$. Let $\mathbb{N}_{0}^{N}$ be the set of all multi-indices $\alpha=\left(\alpha_{1}, \ldots, \alpha_{N}\right)$ and $|\alpha|=\alpha_{1}+\cdots+\alpha_{N}$ be their lengths. Here $\mathbb{N}_{0}=\mathbb{N} \cup\{0\}$. By $W^{m, p}(\Omega), 1 \leq p \leq \infty$, we denote the Sobolev space of all complex-valued functions $u$ in $L^{p}(\Omega)$, which have all weak derivatives $D^{\alpha} u$ up to order $m$ in $L^{p}(\Omega)$, endowed with the norm

$$
\|u\|_{W^{m, p}(\Omega)}=\sum_{|\alpha| \leq m}\left\|D^{\alpha} u\right\|_{L^{p}(\Omega)} .
$$

If $1 \leq p<\infty$, then by $W_{0}^{m, p}(\Omega)$ we denote the closure in $W^{m, p}(\Omega)$ of the space of all $C^{\infty}$-functions with compact support in $\Omega$. For open sets $\Omega$ under consideration a function belongs to $W_{0}^{m, p}(\Omega)$ if and only if its extension by zero outside $\Omega$ belongs to $W^{m, p}\left(\mathbb{R}^{N}\right)$. By $W_{0}^{m, \infty}(\Omega)$ we denote the space of all functions in $W^{m, \infty}(\Omega)$ whose extension by zero outside $\Omega$ belongs to $W^{m, \infty}\left(\mathbb{R}^{N}\right)$, which is wider than the closure in $W^{m, \infty}(\Omega)$ of the space of all $C^{\infty}$-functions with compact support in $\Omega$.

Let $\hat{m}$ be the number of the multi-indices $\alpha \in \mathbb{N}_{0}^{N}$ with $|\alpha|=m$. For all $\alpha, \beta \in \mathbb{N}_{0}^{N}$ such that $|\alpha|=|\beta|=m$, let $A_{\alpha \beta}$ be bounded measurable real-valued functions defined on $\Omega$ such that $A_{\alpha \beta}=A_{\beta \alpha}$ and for some $\theta>0$

$$
\theta^{-1}|\xi|^{2} \leq \sum_{|\alpha|=|\beta|=m} A_{\alpha \beta}(x) \xi_{\alpha} \xi_{\beta} \leq \theta|\xi|^{2}
$$

for all $x \in \Omega, \xi=\left(\xi_{\alpha}\right)_{|\alpha|=m} \in \mathbb{R}^{\hat{m}}$.

Let $V(\Omega)$ be a closed subspace of $W^{m, 2}(\Omega)$ containing $W_{0}^{m, 2}(\Omega)$. We consider the following eigenvalue problem

$$
\int_{\Omega} \sum_{|\alpha|=|\beta|=m} A_{\alpha \beta} D^{\alpha} u D^{\beta} \bar{v} d x=\lambda \int_{\Omega} u \bar{v} d x
$$

for all test functions $v \in V(\Omega)$, in the unknowns $u \in V(\Omega)$ (the eigenfunctions) and $\lambda \in \mathbb{R}$ (the eigenvalues). 
As is well-known, problem (2.3) is the weak formulation of the eigenvalue problem for the operator $H$ in (1.1) subject to suitable homogeneous boundary conditions: the choice of $V(\Omega)$ corresponds to the choice of the boundary conditions (see e.g., Nečas [10]).

We set

$$
Q_{\Omega}(u, v)=\int_{\Omega} \sum_{|\alpha|=|\beta|=m} A_{\alpha \beta} D^{\alpha} u D^{\beta} \bar{v} d x, \quad Q_{\Omega}(u)=Q_{\Omega}(u, u),
$$

for all $u, v \in W^{m, 2}(\Omega)$.

We assume that the embedding $V(\Omega) \subset W^{m-1,2}(\Omega)$ is compact. Then one can prove that the restriction to $V(\Omega)$ of the quadratic form $Q_{\Omega}$ is closed, hence the eigenvalues of equation (2.3) coincide with the eigenvalues of a suitable operator $H_{V(\Omega)}$ canonically associated with $Q_{\Omega}$ and $V(\Omega)$. Since, in particular, the embedding $V(\Omega) \subset L^{2}(\Omega)$ is compact, $H_{V(\Omega)}$ has compact resolvent and the following theorem holds (see [6, Thm. 2.1] for a detailed proof).

Theorem 2.5 Let $\Omega$ be an open set in $\mathbb{R}^{N}$. Let $m \in \mathbb{N}, \theta>0$ and, for all $\alpha, \beta \in \mathbb{N}_{0}^{N}$ such that $|\alpha|=|\beta|=m$, let $A_{\alpha \beta}$ be bounded measurable real-valued functions defined on $\Omega$, satisfying $A_{\alpha \beta}=A_{\beta \alpha}$ and condition (2.2).

Let $V(\Omega)$ be a closed subspace of $W^{m, 2}(\Omega)$ containing $W_{0}^{m, 2}(\Omega)$ and such that the embedding $V(\Omega) \subset W^{m-1,2}(\Omega)$ is compact.

Then there exists a non-negative self-adjoint linear operator $H_{V(\Omega)}$ on $L^{2}(\Omega)$ with compact resolvent, such that $\operatorname{Dom}\left(H_{V(\Omega)}^{1 / 2}\right)=V(\Omega)$ and

$$
<H_{V(\Omega)}^{1 / 2} u, H_{V(\Omega)}^{1 / 2} v>_{L^{2}(\Omega)}=Q_{\Omega}(u, v),
$$

for all $u, v \in V(\Omega)$. Moreover, the eigenvalues of equation (2.3) coincide with the eigenvalues $\lambda_{n}\left[H_{V(\Omega)}\right]$ of $H_{V(\Omega)}$ and

$$
\lambda_{n}\left[H_{V(\Omega)}\right]=\inf _{\substack{\mathcal{L} \subset V(\Omega) \\ \operatorname{dim} \mathcal{L}=n}} \sup _{\substack{u \in \mathcal{L} \\ u \neq 0}} \frac{Q_{\Omega}(u)}{\|u\|_{L^{2}(\Omega)}^{2}},
$$

where the infimum is taken with respect to all subspaces $\mathcal{L}$ of $V(\Omega)$ of dimension $n$.

Note that the compactness of the embedding $V(\Omega) \subset W^{m-1,2}(\Omega)$ can be deduced by the compactness of the embedding $V(\Omega) \subset L^{2}(\Omega)$ under some further assumptions on $\Omega$. Assume that $\Omega$ is such that for any $\epsilon>0$ there exists $c(\epsilon)>0$ such that the following inequality holds:

$$
\|u\|_{W^{m-1,2}(\Omega)} \leq c(\epsilon)\|u\|_{L^{2}(\Omega)}+\epsilon \sum_{|\alpha|=m}\left\|D^{\alpha} u\right\|_{L^{2}(\Omega)} .
$$

(This inequality holds in particular if $\Omega$ has a quasi-continuous boundary, see Burenkov [4, Thm. 6, p. 160].) Then the compactness of the embedding $V(\Omega) \subset$ $L^{2}(\Omega)$ is equivalent to the compactness of the embedding $V(\Omega) \subset W^{m-1,2}(\Omega)$, see Burenkov [4, Lemma 13, p. 172] for details.

In this paper we are interested in the cases $V(\Omega)=W_{0}^{m, 2}(\Omega)$ and $V(\Omega)=$ $W^{m, 2}(\Omega)$ which correspond to Dirichlet and Neumann boundary conditions respectively. 
Definition 2.8 Let $\Omega$ be an open set in $\mathbb{R}^{N}$. Let $m \in \mathbb{N}, \theta>0$ and, for all $\alpha, \beta \in \mathbb{N}_{0}^{N}$ such that $|\alpha|=|\beta|=m$, let $A_{\alpha \beta}$ be bounded measurable real-valued functions defined on $\Omega$, satisfying $A_{\alpha \beta}=A_{\beta \alpha}$ and condition (2.2).

If the embedding $W_{0}^{m, 2}(\Omega) \subset W^{m-1,2}(\Omega)$ is compact, we set

$$
\lambda_{n, \mathcal{D}}[\Omega]=\lambda_{n}\left[H_{W_{0}^{m, 2}(\Omega)}\right] .
$$

If the embedding $W^{m, 2}(\Omega) \subset W^{m-1,2}(\Omega)$ is compact, we set

$$
\lambda_{n, \mathcal{N}}[\Omega]=\lambda_{n}\left[H_{W^{m, 2}(\Omega)}\right] .
$$

The numbers $\lambda_{n, \mathcal{D}}[\Omega], \lambda_{n, \mathcal{N}}[\Omega]$ are called the Dirichlet eigenvalues, Neumann eigenvalues respectively, of operator (1.1).

Remark 2.9 If $\Omega$ is such that the embedding $W_{0}^{1,2}(\Omega) \subset L^{2}(\Omega)$ is compact (for instance, if $\Omega$ is an arbitrary open set with finite Lebesgue measure), then also the embedding $W_{0}^{m, 2}(\Omega) \subset W^{m-1,2}(\Omega)$ is compact and the Dirichlet eigenvalues are well-defined.

If $\Omega$ is such that the embedding $W^{1,2}(\Omega) \subset L^{2}(\Omega)$ is compact (for instance, if $\Omega$ has a continuous boundary, see Definition (2.10), then the embedding $W^{m, 2}(\Omega) \subset$ $W^{m-1,2}(\Omega)$ is compact and the Neumann eigenvalues are well-defined.

In the next sections we shall study the variation of $\lambda_{n, \mathcal{D}}[\Omega]$ and $\lambda_{n, \mathcal{N}}[\Omega]$ upon variation of $\Omega$ in suitable classes of open sets defined below.

For any set $V$ in $\mathbb{R}^{N}$ and $\delta>0$ we denote by $V_{\delta}$ the set $\{x \in V: d(x, \partial \Omega)>\delta\}$. Moreover, as in [5], by a cuboid we mean any rotation of a rectangular parallelepiped in $\mathbb{R}^{N}$.

Definition 2.10 Let $\rho>0, s, s^{\prime} \in \mathbb{N}, s^{\prime} \leq s$ and $\left\{V_{j}\right\}_{j=1}^{s}$ be a family of bounded open cuboids and $\left\{r_{j}\right\}_{j=1}^{s}$ be a family of rotations in $\mathbb{R}^{N}$.

We say that $\mathcal{A}=\left(\rho, s, s^{\prime},\left\{V_{j}\right\}_{j=1}^{s},\left\{r_{j}\right\}_{j=1}^{s}\right)$ is an atlas in $\mathbb{R}^{N}$ with the parameters $\rho, s, s^{\prime},\left\{V_{j}\right\}_{j=1}^{s},\left\{r_{j}\right\}_{j=1}^{s}$, briefly an atlas in $\mathbb{R}^{N}$.

We denote by $C(\mathcal{A})$ the family of all open sets $\Omega$ in $\mathbb{R}^{N}$ satisfying the following properties:

(i) $\Omega \subset \bigcup_{j=1}^{s}\left(V_{j}\right)_{\rho}$ and $\left(V_{j}\right)_{\rho} \cap \Omega \neq \emptyset$;

(ii) $V_{j} \cap \partial \Omega \neq \emptyset$ for $j=1, \ldots s^{\prime}, V_{j} \cap \partial \Omega=\emptyset$ for $s^{\prime}<j \leq s$;

(iii) for $j=1, \ldots, s$

$$
r_{j}\left(V_{j}\right)=\left\{x \in \mathbb{R}^{N}: a_{i j}<x_{i}<b_{i j}, i=1, \ldots, N\right\}
$$

and

$$
r_{j}\left(\Omega \cap V_{j}\right)=\left\{x \in \mathbb{R}^{N}: a_{N j}<x_{N}<g_{j}(\bar{x}), \bar{x} \in W_{j}\right\},
$$

where $\bar{x}=\left(x_{1}, \ldots, x_{N-1}\right), W_{j}=\left\{\bar{x} \in \mathbb{R}^{N-1}: a_{i j}<x_{i}<b_{i j}, i=1, \ldots, N-1\right\}$ and $g_{j}$ is a continuous function defined on $\bar{W}_{j}$ (it is meant that if $s^{\prime}<j \leq s$ then $g_{j}(\bar{x})=b_{N j}$ for all $\left.\bar{x} \in \bar{W}_{j}\right)$; moreover for $j=1, \ldots, s^{\prime}$

$$
a_{N j}+\rho \leq g_{j}(\bar{x}) \leq b_{N j}-\rho,
$$


for all $\bar{x} \in \bar{W}_{j}$.

We say that an open set $\Omega$ in $\mathbb{R}^{N}$ is an open set with a continuous boundary if $\Omega$ is of class $C(\mathcal{A})$ for some atlas $\mathcal{A}$.

Let $m \in \mathbb{N}, M>0$. We say that an open set $\Omega$ is of class $C_{M}^{m}(\mathcal{A}), C_{M}^{m-1,1}(\mathcal{A})$ if $\Omega$ is of class $C(\mathcal{A})$ and all the functions $g_{j}$ in (iii) are of class $C^{m}\left(\bar{W}_{j}\right)$, $C^{m-1,1}\left(\bar{W}_{j}\right)$ with

$$
\begin{gathered}
\left|g_{j}\right|_{c^{m}\left(\bar{W}_{j}\right)}=\sum_{1 \leq|\alpha| \leq m}\left\|D^{\alpha} g_{j}\right\|_{L^{\infty}\left(\bar{W}_{j}\right)} \leq M, \\
\left|g_{j}\right|_{c^{m-1,1}\left(\bar{W}_{j}\right)}=\left|g_{j}\right|_{c^{m-1}\left(\bar{W}_{j}\right)}+\sum_{|\alpha|=m-1} \sup _{\substack{\bar{x}, \bar{y} \in \bar{W}_{j} \\
\bar{x} \neq \bar{y}}} \frac{\left|D^{\alpha} g_{j}(\bar{x})-D^{\alpha} g_{j}(\bar{y})\right|}{|\bar{x}-\bar{y}|} \leq M
\end{gathered}
$$

respectively1.

We say that an open set $\Omega$ in $\mathbb{R}^{N}$ is an open set of class $C^{m}, C^{m-1,1}$ if $\Omega$ is of class $C_{M}^{m}(\mathcal{A}), C_{M}^{m-1,1}(\mathcal{A})$ respectively, for some atlas $\mathcal{A}$ and some $M>0$.

\section{A pre-transition operator for higher order So- bolev spaces}

The aim of this section is proving the following theorem.

Theorem 3.1 Let $\mathcal{A}=\left(\rho, s, s^{\prime},\left\{V_{j}\right\}_{j=1}^{s},\left\{r_{j}\right\}_{j=1}^{s}\right)$ be an atlas in $\mathbb{R}^{N}, m \in \mathbb{N}$, $M>0$. Let $\Omega_{1}, \Omega_{2} \in C_{M}^{m-1,1}(\mathcal{A})$. For all $m \in \mathbb{N}, 1 \leq p \leq \infty$ there exist linear maps

$$
\mathcal{T}_{\mathcal{D}}: W_{0}^{m, p}\left(\Omega_{1}\right) \rightarrow W_{0}^{m, p}\left(\Omega_{2}\right) \text { and } \mathcal{T}_{\mathcal{N}}: W^{m, p}\left(\Omega_{1}\right) \rightarrow W^{m, p}\left(\Omega_{2}\right),
$$

with the following properties:

(i) there exists $C_{1}>0$ depending only on $\mathcal{A}, m, M, p$ such that $\left\|\mathcal{T}_{\mathcal{D}}\right\|,\left\|\mathcal{T}_{\mathcal{N}}\right\| \leq$ $C_{1}$.

(ii) there exists $C_{2}>0$ depending only on $\mathcal{A}$, and an open set $\Omega_{3} \subset \Omega_{1} \cap \Omega_{2}$ such that

$$
\left|\Omega_{1} \backslash \Omega_{3}\right|,\left|\Omega_{2} \backslash \Omega_{3}\right| \leq C_{2}\left|\Omega_{1} \Delta \Omega_{2}\right|
$$

and such that

$$
\mathcal{T}_{\mathcal{D}}[u](x)=u(x), \quad \mathcal{T}_{\mathcal{N}}[v](x)=v(x),
$$

for all $u \in W_{0}^{m, p}\left(\Omega_{1}\right), v \in W^{m, p}\left(\Omega_{2}\right), x \in \Omega_{3}$.

For $m=1$ Theorem 3.1 was proved in [7, 8]. We note that the construction of the operator $\mathcal{T}_{\mathcal{D}}$ in [7] does not have a straightforward generalization to the case $m>1$. A more or less straightforward generalization of a crucial step in the construction in [7] is given in Lemma [3.4, where a special transformation $\Phi_{c}$ is defined depending on a positive constant $c$. Importantly, the derivatives of $\Phi_{c}$ of order greater than one have singularities. This leads to singularities

\footnotetext{
${ }^{1}$ Note that as customary $\left\|g_{j}\right\|_{C^{m}\left(\bar{W}_{j}\right)}=\left\|g_{j}\right\|_{L^{\infty}\left(W_{j}\right)}+\left|g_{j}\right|_{c^{m}\left(\bar{W}_{j}\right)}$ and $\left\|g_{j}\right\|_{C^{m-1,1}\left(\bar{W}_{j}\right)}=$ $\left\|g_{j}\right\|_{L^{\infty}\left(W_{j}\right)}+\left|g_{j}\right|_{c^{m-1,1}\left(\bar{W}_{j}\right)}$.
} 
when applying the chain rule to compositions $v\left(\Phi_{c}\right)$, which are explicitly written out in the first summand of the right-hand side of formula (3.10). In order to overcome this difficulty, in Lemma 3.23 we construct a linear map $T$, given by formula (3.25), with appropriately chosen parameters $\delta_{k}, c_{k}$ which allow to control the effect of singularities and ensure the boundedness of $T$. The proof of the boundedness of $T$ is based on the one-dimensional Lemma 3.19. Finally, in the proof of Theorem 3.1 local transformations of such type are pasted together.

Lemma 3.4 Let $W$ be a bounded convex open set in $\mathbb{R}^{N-1}$. Let $m \in \mathbb{N}, a \in \mathbb{R}$, $D_{1}>D_{2}>a$ and $g_{1}, g_{2} \in C^{m-1,1}(\bar{W})$ be such that

$$
D_{2}<g_{2}(\bar{x}), g_{1}(\bar{x})<D_{1},
$$

for all $\bar{x} \in \bar{W}$. Let $\delta=\frac{D_{2}-a}{2\left(D_{1}-D_{2}\right)}$ and $c \geq \frac{1}{\delta}$. Let

$$
\begin{aligned}
& g_{3}(\bar{x})=g_{2}(\bar{x})-\delta\left(g_{1}(\bar{x})-g_{2}(\bar{x})\right)^{+} \\
& g_{1, c}(\bar{x})=g_{2}(\bar{x})+c \delta\left(g_{1}(\bar{x})-g_{2}(\bar{x})\right)^{+},
\end{aligned}
$$

for all $\bar{x} \in \bar{W}$, and let

$$
\begin{aligned}
& \mathcal{O}_{k}=\left\{\left(\bar{x}, x_{N}\right): \bar{x} \in W, a<x_{N}<g_{k}(\bar{x})\right\}, \quad k=1,2,3, \\
& \mathcal{O}_{1, c}=\left\{\left(\bar{x}, x_{N}\right): \bar{x} \in W, a<x_{N}<g_{1, c}(\bar{x})\right\} .
\end{aligned}
$$

Let $\Phi_{c}$ be the map of $\overline{\mathcal{O}}_{2}$ into $\overline{\mathcal{O}}_{1 c}$ defined by

$$
\Phi_{c}(x)=\left(\bar{x}, x_{N}+\operatorname{ch}(x)\right), \quad x \in \overline{\mathcal{O}}_{2}
$$

where

$$
h(x)= \begin{cases}0, & \text { if } x \in \overline{\mathcal{O}}_{3}, \\ \frac{\left(x_{N}-g_{3}(\bar{x})\right)^{m+1}}{\delta^{m}\left(g_{1}(\bar{x})-g_{2}(\bar{x})\right)^{m}}, & \text { if } x \in \overline{\mathcal{O}}_{2} \backslash \overline{\mathcal{O}}_{3},\end{cases}
$$

Then the following statements hold:

(i) $\emptyset \neq \mathcal{O}_{3} \subset \mathcal{O}_{2} ; \mathcal{O}_{1}, \mathcal{O}_{2}, \mathcal{O}_{3} \subset \mathcal{O}_{1, c}$ and $\left|\mathcal{O}_{1, c} \backslash \mathcal{O}_{2}\right|=c \delta\left|\mathcal{O}_{1} \backslash \mathcal{O}_{2}\right|=c\left|\mathcal{O}_{2} \backslash \mathcal{O}_{3}\right|$;

(ii) $\Phi_{c}$ is a bijection of $\overline{\mathcal{O}}_{2}$ onto $\overline{\mathcal{O}}_{1, c}, \Phi_{c}\left(\partial \mathcal{O}_{2}\right)=\partial \mathcal{O}_{1, c}, \Phi_{c} \in C_{l o c}^{m-1,1}\left(\mathcal{O}_{2}\right) \cap$ $\operatorname{Lip}\left(\mathcal{O}_{2}\right)$, and $\Phi_{c}(x)=x$ for all $x \in \mathcal{O}_{3}$;

(iii) there exists $M>0$ depending only on $N, m, a, D_{1}, D_{2},\left\|g_{1}\right\|_{c^{m-1,1}(\bar{W})}$ and $\left\|g_{2}\right\|_{c^{m-1,1}(\bar{W})}$ such that for all $\alpha \in \mathbb{N}_{0}^{N}$ with $|\alpha| \leq m$

$$
\left\|h^{|\alpha|-1} D^{\alpha} h\right\|_{L^{\infty}\left(\mathcal{O}_{2} \backslash \overline{\mathcal{O}}_{3}\right)} \leq M
$$

Proof. We note that if $\bar{x} \in \bar{W}$ and $g_{2}(\bar{x}) \geq g_{1}(\bar{x})$ then $g_{3}(\bar{x})=g_{1, c}(\bar{x})=g_{2}(\bar{x})$; viceversa, if $g_{2}(\bar{x})<g_{1}(\bar{x})$, since $c \delta \geq 1$ it follows that $g_{3}(\bar{x})<g_{2}(\bar{x})<g_{1}(\bar{x})<$ $g_{1, c}(\bar{x})$. In particular, $\mathcal{O}_{3} \subset \mathcal{O}_{2}$ and $\mathcal{O}_{1}, \mathcal{O}_{2}, \mathcal{O}_{3} \subset \mathcal{O}_{1, c} ;$ moreover, if $\left(\bar{x}, x_{N}\right) \in$ $\overline{\mathcal{O}}_{2} \backslash \overline{\mathcal{O}}_{3}$ then $g_{2}(\bar{x})<g_{1}(\bar{x})$, hence $\Phi_{c}$ is well defined. Since $\delta<\left(D_{2}-a\right) /\left(D_{1}-D_{2}\right)$ then $a<g_{3}(\bar{x})$ for all $\bar{x} \in \bar{W}$, hence $\mathcal{O}_{3} \neq \emptyset$. Moreover, we note that

$$
g_{1, c}(\bar{x})-g_{2}(\bar{x})=c \delta\left(g_{1}(\bar{x})-g_{2}(\bar{x})\right)^{+}=c\left(g_{2}(\bar{x})-g_{3}(\bar{x})\right),
$$

hence the equalities in statement (i) follow. 
Statement (iii) follows by standard calculus.

We now prove statement (ii). By using the same argument as in [7, Lemma 4.1] one can prove that $\Phi_{c} \in \operatorname{Lip}\left(\mathcal{O}_{2}\right)$. Moreover, it is obvious that $\Phi_{c}$ is a bijection of $\overline{\mathcal{O}}_{2}$ onto $\overline{\mathcal{O}}_{1, c}$ and $\Phi_{c}\left(\partial \mathcal{O}_{2}\right)=\partial \mathcal{O}_{1, c}$.

It remains to prove that $\Phi_{c} \in C_{l o c}^{m-1,1}\left(\mathcal{O}_{2}\right)$. Clearly $\Phi_{c}$ is of class $C_{l o c}^{m-1,1}$ on the open sets $\mathcal{O}_{3}$ and $\mathcal{O}_{2} \backslash \overline{\mathcal{O}}_{3}$. We now prove that $\Phi_{c}$ is of class $C^{m-1}$ in a neighborhood of any point of $\mathcal{O}_{2} \cap \partial \mathcal{O}_{3}$. It clearly suffices to do so for $\left(\Phi_{c}\right)_{N}$. Let $\left(\bar{y}, y_{N}\right) \in \mathcal{O}_{2} \cap \partial \mathcal{O}_{3}$. Then $y_{N}=g_{3}(\bar{y})<g_{2}(\bar{y})<g_{1}(\bar{y})$ and by continuity there exists an open neighborhood $U$ of $\left(\bar{y}, y_{N}\right)$ contained in $\mathcal{O}_{2}$ such that $g_{3}(\bar{x})<g_{2}(\bar{x})<g_{1}(\bar{x})$ for all $\left(\bar{x}, x_{N}\right) \in U$.

Consider the functions $\varphi_{1}\left(\bar{x}, x_{N}\right)=x_{N}$ and $\varphi_{2}\left(\bar{x}, x_{N}\right)=x_{N}+\frac{c\left|x_{N}-g_{3}(\bar{x})\right|^{m+1}}{\delta^{m}\left(g_{1}(\bar{x})-g_{2}(\bar{x})\right)^{m}}$ for all $\left(\bar{x}, x_{N}\right) \in U$. Clearly $\varphi_{1}, \varphi_{2} \in C^{m-1,1}(U)$ and $D^{\alpha} \varphi_{1}=D^{\alpha} \varphi_{2}$ on $U \cap \partial \mathcal{O}_{3}$ for all $\alpha \in \mathbb{N}_{0}^{N}$ with $|\alpha| \leq m-1$. Since $\left(\Phi_{c}\right)_{N}=\varphi_{1}$ on $U \cap \overline{\mathcal{O}}_{3}$ and $\left(\Phi_{c}\right)_{N}=\varphi_{2}$ on $U \backslash \overline{\mathcal{O}}_{3}$ it follows that $\left(\Phi_{c}\right)_{N} \in C^{m-1}(U)$. Moreover, since $D^{\alpha} \varphi_{1}=D^{\alpha} \varphi_{2}$ on the graph of $g_{3}$ for all $|\alpha|=m-1$, it follows that $D^{\alpha}\left(\Phi_{c}\right)_{N}$, hence $D^{\alpha} \Phi_{c}$ is locally Lipschitz continuous on $\mathcal{O}_{2}$ for all $|\alpha|=m-1$.

Lemma 3.9 Let the assumptions of Lemma 3.4 hold. If $v \in W_{l o c}^{m, 1}\left(\mathcal{O}_{1, c}\right)$ then $v \circ \Phi_{c} \in W_{l o c}^{m, 1}\left(\mathcal{O}_{2}\right)$ and for each $\alpha \in \mathbb{N}_{0}^{N}$ with $1 \leq|\alpha| \leq m$

$$
\begin{aligned}
& D^{\alpha}\left(v\left(\Phi_{c}\right)\right)(x) \\
& \quad=\sum_{1 \leq|\beta|<|\alpha|} \frac{\left(D^{\beta} v\right)\left(\Phi_{c}(x)\right)}{h(x)^{|\alpha|-|\beta|}} \sum_{r=1}^{|\beta|} b_{\beta, r}(x) c^{r}+\sum_{|\beta|=|\alpha|}\left(D^{\beta} v\right)\left(\Phi_{c}(x)\right) \sum_{r=0}^{|\beta|} b_{\beta, r}(x) c^{r},
\end{aligned}
$$

for all $x \in \mathcal{O}_{2} \backslash \overline{\mathcal{O}}_{3}$, where $b_{\beta, r}$ are bounded continuous functions independent of c. Moreover, there exists $M>0$ depending only on $N, m, a, D_{1}, D_{2},\left\|g_{1}\right\|_{c^{m-1,1}(\bar{W})}$ and $\left\|g_{2}\right\|_{c^{m-1,1}(\bar{W})}$ such that all functions $b_{\beta, r}$ in (3.10) satisfy the inequality

$$
\left\|b_{\beta, r}\right\|_{L^{\infty}\left(\mathcal{O}_{2} \backslash \overline{\mathcal{O}}_{3}\right)} \leq M
$$

Proof. If $\phi=\left(\phi_{1}, \ldots, \phi_{N}\right)$ is a map of $\mathcal{O}_{2}$ to $\mathcal{O}_{1, c}$ of class $C_{l o c}^{m-1,1}$ then $v \circ \phi \in W_{l o c}^{m, 1}\left(\mathcal{O}_{2}\right)$ for all $v \in W_{l o c}^{m, 1}\left(\mathcal{O}_{1, c}\right)$. Moreover, by the chain rule $D^{\alpha}(v(\phi))$ is a linear combination of the functions

$$
\left(D^{\beta} v\right)(\phi) D^{\nu_{i_{1}}} \phi_{i_{1}} \cdots D^{\nu_{i_{k}}} \phi_{i_{k}}
$$

with natural coefficients depending only on $\alpha, \beta, \nu_{i_{1}}, \ldots, \nu_{i_{k}}$, where $1 \leq|\beta| \leq|\alpha|$, $k=|\beta|, i_{1}, \ldots i_{k} \in\{1, \ldots, N\}, \nu_{i_{1}}, \ldots, \nu_{i_{k}} \in \mathbb{N}_{0}^{N}$, and

$$
\left|\nu_{i_{1}}\right|+\cdots+\left|\nu_{i_{k}}\right|=|\alpha|, \quad\left|\nu_{i_{1}}\right|, \ldots,\left|\nu_{i_{k}}\right| \geq 1
$$

In particular if $\phi=\Phi_{c}$ then $\phi_{i}(x)=x_{i}$ for all $i=1, \ldots, N-1$, and $\phi_{N}(x)=$ $x_{N}+\operatorname{ch}(x)$ for all $x \in \mathcal{O}_{2}$, where $h$ defined by (3.8). If $i_{1}, \ldots, i_{k} \in\{1, \ldots, N-$ $1\}$ then among the functions in (3.11) we can consider only those with $\nu_{i_{1}}=$ $e_{i_{1}}, \ldots, \nu_{i_{k}}=e_{i_{k}}$, (here $e_{1}, \ldots, e_{N}$ denotes the canonical basis in $\mathbb{R}^{N}$ ) in which case $|\beta|=|\alpha|$ by (3.12): thus, in this case we can consider only functions of the type

$$
\left(D^{\beta} v\right)\left(\Phi_{c}\right)
$$


with $|\beta|=|\alpha|$. The remaining functions correspond to the cases when at least one of the indices $i_{s}$ is $N$. Assume that exactly $n$ of them are equal to $N$, then $\nu_{i_{s}}=e_{i_{s}}$ for the remaining $|\beta|-n$ of them. Thus, such functions are of the type

$$
\left(D^{\beta} v\right)\left(\Phi_{c}\right) D^{\eta_{1}}\left(x_{N}+\operatorname{ch}(x)\right) \cdots D^{\eta_{n}}\left(x_{N}+\operatorname{ch}(x)\right)
$$

where $1 \leq|\beta| \leq|\alpha|, 1 \leq n \leq|\beta|, \eta_{1}, \ldots, \eta_{n} \in \mathbb{N}_{0}^{N}$, and

$$
\left|\eta_{1}\right|+\cdots+\left|\eta_{n}\right|=|\alpha|-|\beta|+n, \quad\left|\eta_{1}\right|, \ldots,\left|\eta_{n}\right| \geq 1
$$

The functions in (3.14) are linear combinations of functions of the type

$$
c^{\rho}\left(D^{\beta} v\right)\left(\Phi_{c}\right) D^{\xi_{1}} h \cdots D^{\xi_{\rho}} h
$$

with natural coefficients depending only on $\alpha, \beta, \rho, \xi_{1}, \ldots, \xi_{\rho}$, where

$$
1 \leq \rho \leq n, \quad\left|\xi_{1}\right|+\cdots+\left|\xi_{\rho}\right|=|\alpha|-|\beta|+\rho, \quad\left|\xi_{1}\right|, \ldots,\left|\xi_{n}\right| \geq 1
$$

and of functions of the type (3.13) which correspond to the case $\eta_{1}=\cdots=\eta_{n}=$ $e_{N}$, in which case $|\alpha|=|\beta|$ by (3.15).

By Lemma 3.4 the functions $b_{\xi_{s}}=h^{\left|\xi_{s}\right|-1} D^{\xi_{s}} h$ are continuous, bounded and such that $\left\|b_{\xi_{s}}\right\|_{L^{\infty}\left(\mathcal{O}_{2} \backslash \overline{\mathcal{O}}_{3}\right)} \leq M_{1}$ where $M_{1}$ is constant depending only on $N, m, a$, $D_{1}, D_{2},\left\|g_{1}\right\|_{c^{m-1,1}(\bar{W})}$ and $\left\|g_{2}\right\|_{c^{m-1,1}(\bar{W})}$. Hence

$$
\begin{aligned}
& c^{\rho}\left(D^{\beta} v\right)\left(\Phi_{c}\right) D^{\xi_{1}} h \cdots D^{\xi_{\rho}} h \\
& \quad=c^{\rho}\left(D^{\beta} v\right)\left(\Phi_{c}\right) b_{\xi_{1}} \cdots b_{\xi_{\rho}} h^{\rho-\left(\left|\xi_{1}\right|+\cdots+\left|\xi_{\rho}\right|\right)}=c^{\rho}\left(D^{\beta} v\right)\left(\Phi_{c}\right) b_{\xi_{1}} \cdots b_{\xi_{\rho}} h^{|\beta|-|\alpha|} .
\end{aligned}
$$

Thus $D^{\alpha}\left(v\left(\Phi_{c}\right)\right)$ is a linear combination with natural coefficients depending only on $\alpha, \beta, \rho, \xi_{1}, \ldots, \xi_{\rho}$ of functions of the type (3.13) with $|\beta|=|\alpha|$ and of the type (3.18) with $1 \leq|\beta| \leq|\alpha|$ and $1 \leq \rho \leq|\beta|$. Clearly, such functions can be arranged as in formula (3.10).

Lemma 3.19 Let $\mu, s \in \mathbb{N}, s \leq \mu, 0<c_{1}<\cdots<c_{\mu}, \gamma_{1}, \ldots, \gamma_{\mu} \in \mathbb{R}$ and

$$
\sum_{k=1}^{\mu} \gamma_{k} c_{k}^{\sigma}=0, \quad \sigma=1, \ldots, s
$$

Moreover, let $-\infty<a<b<\infty, \eta \in C^{1}[a, b]$ and $\eta(x)>0, \eta^{\prime}(x) \geq 0$ for all $x \in(a, b)$. Then

$$
\left\|\eta^{-s}(x) \sum_{k=1}^{\mu} \gamma_{k} c_{k} f\left(x+c_{k} \eta(x)\right)\right\|_{L^{p}(a, b)} \leq C\left\|f^{(s)}\right\|_{L^{p}\left(a, b+c_{\mu} \eta(b)\right)},
$$

for all $1 \leq p \leq \infty$ and for all $f \in W^{s, p}\left(a, b+c_{\mu} \eta(b)\right)$, where

$$
C=\sum_{k=1}^{\mu}\left|\gamma_{k}\right| c_{k}\left(c_{k}-c_{1}\right)^{s}
$$


Proof. If $\left.f \in W^{s, p}\left(a, b+c_{\mu} \eta(b)\right)\right)$ there exists a function $g$ equivalent to $f$ on $\left(a, b+c_{\mu} \eta(b)\right)$ whose derivative $g^{(s-1)}$ is absolutely continuous on $\left[a, b+c_{\mu} \eta(b)\right]$.

By (3.20) for all $x \in(a, b)$

$$
\begin{aligned}
& \sum_{k=1}^{\mu} \gamma_{k} c_{k} g\left(x+c_{k} \eta(x)\right)=\sum_{k=1}^{\mu} \gamma_{k} c_{k}\left(g\left(x+c_{k} \eta(x)\right)-g\left(x+c_{1} \eta(x)\right)\right) \\
& =\eta(x) \sum_{k=1}^{\mu} \gamma_{k} c_{k}\left(c_{k}-c_{1}\right) \int_{0}^{1} g^{\prime}\left(x+c_{1} \eta(x)+t_{1}\left(c_{k}-c_{1}\right) \eta(x)\right) d t_{1} \\
& =\eta(x) \sum_{k=1}^{\mu} \gamma_{k} c_{k}\left(c_{k}-c_{1}\right) \int_{0}^{1} g^{\prime}\left(x+c_{1} \eta(x)+t_{1}\left(c_{k}-c_{1}\right) \eta(x)\right)-g^{\prime}\left(x+c_{1} \eta(x)\right) d t_{1} \\
& =\eta^{2}(x) \sum_{k=1}^{\mu} \gamma_{k} c_{k}\left(c_{k}-c_{1}\right)^{2} \int_{0}^{1} \int_{0}^{1} t_{1} g^{\prime \prime}\left(x+c_{1} \eta(x)+t_{1} t_{2}\left(c_{k}-c_{1}\right) \eta(x)\right) d t_{2} d t_{1}=\ldots \\
& =\eta^{s}(x) \sum_{k=1}^{\mu} \gamma_{k} c_{k}\left(c_{k}-c_{1}\right)^{s} \int_{0}^{1} \ldots \int_{0}^{1} t_{1} \cdots t_{s-1} g^{(s)}\left(x+c_{1} \eta(x)+t_{1} \cdots t_{s}\left(c_{k}-c_{1}\right) \eta(x)\right) d t_{s} \ldots d t_{1} .
\end{aligned}
$$

By Minkowski's inequality for integrals

$$
\begin{aligned}
& \left\|\eta^{-s}(x) \sum_{k=1}^{\mu} \gamma_{k} c_{k} f\left(x+c_{k} \eta(x)\right)\right\|_{L^{p}(a, b)}=\left\|\eta^{-s}(x) \sum_{k=1}^{\mu} \gamma_{k} c_{k} g\left(x+c_{k} \eta(x)\right)\right\|_{L^{p}(a, b)} \\
& \quad \leq \sum_{k=1}^{\mu}\left|\gamma_{k}\right| c_{k}\left(c_{k}-c_{1}\right)^{s} \int_{0}^{1} \ldots \int_{0}^{1}\left\|g^{(s)}\left(x+c_{1} \eta(x)+t_{1} \cdots t_{s}\left(c_{k}-c_{1}\right) \eta(x)\right)\right\|_{L^{p}(a, b)} d t_{s} \ldots d t_{1} .
\end{aligned}
$$

Let $y=x+c_{1} \eta(x)+t_{1} \cdots t_{s}\left(c_{k}-c_{1}\right) \eta(x)$. Note that for all $x \in[a, b], a \leq y(x) \leq$ $b+c_{\mu} \eta(b)$ and $y^{\prime}(x) \geq 1$. Hence

$$
\begin{aligned}
\| g^{(s)}\left(x+c_{1} \eta(x)+t_{1}\right. & \left.\cdots t_{s}\left(c_{k}-c_{1}\right) \eta(x)\right) \|_{L^{p}(a, b)} \\
& \leq\left\|g^{(s)}(y)\right\|_{L^{p}\left(a, b+c_{\mu} \eta(b)\right)}=\left\|f^{(s)}\right\|_{L^{p}\left(a, b+c_{\mu} \eta(b)\right)}
\end{aligned}
$$

and the statement follows.

Lemma 3.23 Let $W, m, a, D_{1}, D_{2}, \delta, g_{1}, g_{2}$ be as in Lemma 3.4. Let $\delta_{1}, \ldots, \delta_{m} \in$ $\mathbb{R}, 1 / \delta \leq c_{1}<\cdots<c_{m}$ be such that

$$
\sum_{k=1}^{m} \delta_{k}=1, \quad \text { and } \quad \sum_{k=1}^{m} \delta_{k} c_{k}^{\tau}=0, \quad \tau=1, \ldots, m-1 .
$$

Let $g_{3}, g_{c_{k}}, \mathcal{O}_{1}, \mathcal{O}_{2}, \mathcal{O}_{3}, \mathcal{O}_{1, c_{k}}, \Phi_{c_{k}}$ be as in Lemma 3.4 with c replaced by $c_{k}$ for all $k=1, \ldots, m$. Let $T$ be the linear map of $L_{l o c}^{1}(W \times] a, \infty[)$ to $L_{l o c}^{1}\left(\mathcal{O}_{2}\right)$ defined by

$$
T[v](x)=\sum_{k=1}^{m} \delta_{k} v\left(\Phi_{c_{k}}(x)\right),
$$

for all $x \in \mathcal{O}_{2}$ and for all $v \in L_{l o c}^{1}(W \times] a, \infty[)$. 
Then for all $1 \leq p \leq \infty$

$$
T: W^{m, p}(W \times] a, \infty[) \rightarrow W^{m, p}\left(\mathcal{O}_{2}\right)
$$

and there exists $C>0$ depending only on $N, m, p, a, D_{1}, D_{2}, \delta, c_{m},\left\|g_{1}\right\|_{c^{m-1,1}(\bar{W})}$, $\left\|g_{2}\right\|_{c^{m-1,1}(\bar{W})}$ such that $\|T\| \leq C$.

Moreover, $T[v](x)=v(x)$ for all $v \in L_{l o c}^{1}(W \times] a, \infty[), x \in \mathcal{O}_{3}$, and if $v=0$ on $\mathcal{O}_{1}^{c}$ then $T[v]=0$ on $\mathcal{O}_{2}^{c}$.

Proof. First of all we recall that if $x \in \mathcal{O}_{3}$ then $\Phi_{c_{k}}(x)=x$ for all $k=$ $1, \ldots, m$. Thus by the first condition in (3.24) it follows that

$$
T[v](x)=\sum_{k=1}^{m} \delta_{k} v\left(\Phi_{c_{k}}(x)\right)=\sum_{k=1}^{m} \delta_{k} v(x)=v(x),
$$

for all $v \in L_{l o c}^{1}(W \times] a, \infty[), x \in \mathcal{O}_{3}$.

Let $v \in W^{m, p}(W \times] a, \infty[)$. By Lemma $3.9 T[v] \in W_{l o c}^{m, 1}\left(\mathcal{O}_{2}\right)$ and

$$
D^{\alpha}(T[v](x))=\sum_{1 \leq|\beta| \leq|\alpha|} \sum_{r=0}^{|\beta|} \frac{b_{\beta, r}(x)}{h(x)^{|\alpha|-|\beta|}} \sum_{k=1}^{m} \delta_{k} c_{k}^{r}\left(D^{\beta} v\right)\left(\bar{x}, x_{N}+c_{k} h(x)\right)
$$

for all $|\alpha|=m$ and for all $x \in \mathcal{O}_{2} \backslash \overline{\mathcal{O}}_{3}$, where $b_{\beta, 0}=0$ if $|\beta|<|\alpha|$. We now estimate the $L^{p}$ norms of the summonds in the right-hand side of (3.28). We consider first the case $1 \leq p<\infty,|\beta|<|\alpha|, 1 \leq r \leq|\beta|$. In this case we apply Lemma 3.19 with $f\left(x_{N}\right)=D^{\beta} v\left(\bar{x}, x_{N}\right), a=g_{3}(\bar{x}), b=g_{2}(\bar{x}), \eta\left(x_{N}\right)=h\left(\bar{x}, x_{N}\right)$, $\mu=m, \gamma_{k}=\delta_{k} c_{k}^{r-1}, s=|\alpha|-|\beta|$.

Note that by (3.24)

$$
\sum_{k=1}^{m} \gamma_{k} c_{k}^{\sigma}=\sum_{k=1}^{m} \delta_{k} c_{k}^{\sigma+r-1}=0, \quad \sigma=1, \ldots,|\alpha|-|\beta| .
$$

Indeed, $1 \leq r \leq|\beta|$, hence $1 \leq \sigma+r-1 \leq|\alpha|-1 \leq m-1$ for all $\sigma=1, \ldots,|\alpha|-|\beta|$. Thus, condition (3.20) is satisfied and by Lemma 3.19 we have

$$
\begin{aligned}
& \left(\int_{W} \int_{g_{3}(\bar{x})}^{g_{2}(\bar{x})}\left|\frac{1}{h(x)^{|\alpha|-|\beta|}} \sum_{k=1}^{m} \delta_{k} c_{k}^{r}\left(D^{\beta} v\right)\left(\bar{x}, x_{N}+c_{k} h(x)\right)\right|^{p} d x_{N} d \bar{x}\right)^{1 / p} \\
& \leq \tilde{c}_{1}\left(\int_{W} \int_{a}^{\infty}\left|D^{\left(\bar{\beta}, \beta_{N}+|\alpha|-|\beta|\right)} v\left(\bar{x}, x_{N}\right)\right|^{p} d x_{N} d \bar{x}\right)^{1 / p} \leq \tilde{c}_{1}\|v\|_{W^{m, p}(W \times] a, \infty[)}
\end{aligned}
$$

where $\beta=\left(\bar{\beta}, \beta_{N}\right)$. In the case $1 \leq p<\infty,|\beta|=|\alpha|, 0 \leq r \leq|\beta|$, by a simple change of variables we obtain

$$
\begin{aligned}
& \left(\int_{W} \int_{g_{3}(\bar{x})}^{g_{2}(\bar{x})}\left|\sum_{k=0}^{m} \delta_{k} c_{k}^{r} \frac{\left(D^{\beta} v\right)\left(\bar{x}, x_{N}+c_{k} h(x)\right)}{h(x)^{|\alpha|-|\beta|}}\right|^{p} d x_{N} d \bar{x}\right)^{1 / p} \\
& \quad \leq\left(\int_{W} \int_{a}^{\infty}\left|\sum_{k=0}^{m} \delta_{k} c_{k}^{r}\left(D^{\beta} v\right)\left(\bar{x}, x_{N}+c_{k} h(x)\right)\right|^{p} d x_{N} d \bar{x}\right)^{1 / p} \leq \tilde{c}_{2}\|v\|_{W^{m, p}(W \times] a, \infty[)} .
\end{aligned}
$$


Thus by (3.28), (3.29), (3.30)

$$
\left\|D^{\alpha} T[v]\right\|_{L^{p}\left(\mathcal{O}_{2} \backslash \mathcal{O}_{3}\right)} \leq \tilde{c}_{3}\|v\|_{W^{m, p}(W \times] a, \infty[)},
$$

for all $v \in W^{m, p}(W \times] a, \infty[)$. Clearly, by (3.27)

$$
\left\|D^{\alpha} T[v]\right\|_{L^{p}\left(\mathcal{O}_{3}\right)}=\left\|D^{\alpha} v\right\|_{L^{p}\left(\mathcal{O}_{3}\right)} \leq\|v\|_{W^{m, p}(W \times] a, \infty[)},
$$

Thus

$$
\|T[v]\|_{W^{m, p}\left(\mathcal{O}_{2}\right)} \leq \tilde{c}_{4}\|v\|_{W^{m, p}(W \times] a, \infty[)} .
$$

for all $v \in W^{m, p}(W \times] a, \infty[)$. In (3.29)-(3.32), $\tilde{c}_{1}, \tilde{c}_{2}, \tilde{c}_{3}, \tilde{c}_{4}$ are constants which clearly can be estimated above by a constant depending only on $N, m, p, a, D_{1}, D_{2}$, $\delta, c_{m},\left\|g_{1}\right\|_{c^{m-1,1}(\bar{W})},\left\|g_{2}\right\|_{c^{m-1,1}(\bar{W})}$. Thus, $T$ maps $W^{m, p}(W \times] a, \infty[)$ to $W^{m, p}\left(\mathcal{O}_{2}\right)$, and is a linear and continuous map with $\|T\|$ as in the statement.

The argument above works also for the case $p=\infty$ provided that integrals are replaced by the corresponding $L^{\infty}$ norms.

Finally, if $v \in L_{l o c}^{1}(W \times] a, \infty[)$ is such that $v=0$ on $\mathcal{O}_{1}^{c}$ then $v=0$ on $\mathcal{O}_{1, c_{k}}^{c}$ hence $v\left(\Phi_{c_{k}}\right)=0$ on $\mathcal{O}_{2}^{c}$ for all $k=1, \ldots, m$; thus $T[v]=0$ on $\mathcal{O}_{2}^{c}$.

Proof of Theorem 3.1. We divide the proof into three steps.

Step 1. Recall that $r_{j}\left(V_{j}\right)$ is a cuboid $\left.\Pi_{k=1}^{N}\right] a_{k j}, b_{k j}[$ and

$$
r_{j}\left(\Omega_{i} \cap V_{j}\right)=\left\{\left(\bar{x}, x_{N}\right) \in \mathbb{R}^{N}: \bar{x} \in \Pi_{k=1}^{N-1}\right] a_{k j}, b_{k j}\left[, a_{N j}<x_{N}<g_{i, j}(\bar{x})\right\},
$$

for $i=1,2$, where $g_{i, j} \in C^{m-1,1}\left(\bar{W}_{j}\right)$ and

$$
a_{N j}+\frac{\rho}{2}<g_{2, j}(\bar{x}), g_{1, j}(\bar{x})<b_{N j}-\frac{\rho}{2},
$$

for all $j=1, \ldots, s^{\prime}, \bar{x} \in \bar{W}_{j}$.

For each $j=1, \ldots, s^{\prime}$ we apply Lemma 3.23, with $W=W_{j}, a=a_{N j}, D_{1}=$ $b_{N j}-\rho / 2, D_{2}=a_{N j}+\rho / 2, g_{1}=g_{1, j}, g_{2}=g_{2, j}$, hence

$$
\delta=\frac{1}{2} \min _{j=1, \ldots, s^{\prime}} \frac{\rho}{2\left(b_{N j}-a_{N j}-\rho\right)},
$$

and with $c_{k}=k-1+1 / \delta$, for all $k=1, \ldots, m$, and $\delta_{k}$ determined by (3.24). Accordingly, for each $j=1, \ldots, s^{\prime}$, we consider the sets $\mathcal{O}_{1}=\mathcal{O}_{1, j}, \mathcal{O}_{2}=\mathcal{O}_{2, j}$, $\mathcal{O}_{3}=\mathcal{O}_{3, j}$ defined by (3.6) and the map $T=T_{j}, T_{j}: L_{l o c}^{1}\left(W_{j} \times\right] a_{N j}, \infty[) \rightarrow$ $L_{\text {loc }}^{1}\left(\mathcal{O}_{2, j}\right)$ defined by (3.25). Observe that $\mathcal{O}_{i, j}=r_{j}\left(\Omega_{i} \cap V_{j}\right), i=1,2$. Finally, for all $j=1, \ldots, s^{\prime}$, we set

$$
\mathcal{T}_{j}[v] \equiv\left(T_{j}\left[\left(v \circ r_{j}^{(-1)}\right)_{\left.\left.\right|_{\left.W_{j} \times\right] a_{N j}, \infty}\right]}\right]\right) \circ r_{j}
$$

for all $v \in L_{l o c}^{1}\left(\mathbb{R}^{N}\right)$, and

$$
\Omega_{3, j} \equiv r_{j}^{(-1)}\left(\mathcal{O}_{3, j}\right)
$$

By Lemma 3.23 it follows that $\mathcal{T}_{j}: L_{l o c}^{1}\left(\mathbb{R}^{N}\right) \rightarrow L_{l o c}^{1}\left(\Omega_{2} \cap V_{j}\right), \mathcal{T}_{j}: W^{m, p}\left(\mathbb{R}^{N}\right) \rightarrow$ $W^{m, p}\left(\Omega_{2} \cap V_{j}\right)$, for all $1 \leq p \leq \infty, j=1, \ldots, s^{\prime}$. Moreover, $\mathcal{T}_{j}[v](x)=v(x)$ for all $v \in L_{l o c}^{1}\left(\mathbb{R}^{N}\right), x \in \Omega_{3, j}$, and there exists $C_{1, j}>0$ depending only on $N, m, \rho, a_{N j}, b_{N j}, M$ and there exists $C_{2, j}>0$ depending only on $\rho, a_{N j}, b_{N j}$ such 
that $\left\|\mathcal{T}_{j}\right\| \leq C_{1, j}$ and $\left|\left(\Omega_{2} \cap V_{j}\right) \backslash \Omega_{3, j}\right| \leq C_{2, j}\left|\left(\Omega_{1} \cap V_{j}\right) \backslash\left(\Omega_{2} \cap V_{j}\right)\right|$. Furthermore, if $v \in L_{l o c}^{1}\left(\mathbb{R}^{N}\right)$ and $v=0$ on $\left(\Omega_{1} \cap V_{j}\right)^{c}$ then $\mathcal{T}_{j} v=0$ on $\left(\Omega_{2} \cap V_{j}\right)^{c}$.

Step 2. We paste together the functions $\mathcal{T}_{j}$ defined in Step 1. To do so, we consider a partition of unity $\left\{\psi_{j}\right\}_{j=1}^{s}$ such that $\psi_{j} \in C_{c}^{\infty}\left(\left(V_{j}\right)_{\frac{3}{4} \rho}\right)$ for all $j=$ $1, \ldots, s$ and such that $\sum_{j=1}^{s} \psi_{j}(x)=1,0 \leq \psi_{j}(x) \leq 1$ and $\left|\nabla \psi_{j}(x)\right| \leq C_{3}$ for all $x \in \cup_{j=1}^{s}\left(V_{j}\right)_{\rho}$, where $C_{3}$ depends only on $\mathcal{A}$.

For all $j=1, \ldots, s^{\prime}$, let $\mathcal{T}_{j}: L_{\text {loc }}^{1}\left(\mathbb{R}^{N}\right) \rightarrow L_{\text {loc }}^{1}\left(\Omega_{2} \cap V_{j}\right)$ be as in Step 1, and for all $s^{\prime}<j \leq s$, let $\mathcal{T}_{j}$ be the restriction operator from $L_{\text {loc }}^{1}\left(\mathbb{R}^{N}\right)$ to $L_{l o c}^{1}\left(V_{j}\right)$. Then we consider the operator $\mathcal{T}$ of $L_{l o c}^{1}\left(\mathbb{R}^{N}\right)$ to $L_{l o c}^{1}\left(\Omega_{2}\right)$ which takes $v \in L_{l o c}^{1}\left(\mathbb{R}^{N}\right)$ to

$$
\mathcal{T}[v]=\sum_{j=1}^{s} \mathcal{T}_{j}\left[\psi_{j} v\right],
$$

for all $v \in L_{\text {loc }}^{1}\left(\mathbb{R}^{N}\right)$. Clearly, if $v \in W^{m, p}\left(\mathbb{R}^{N}\right)$ then $\mathcal{T}_{j}\left[\psi_{j} v\right] \in W^{m, p}\left(\Omega_{2}\right)$.

Step 3. Since $\Omega_{1} \in C_{M}^{m-1,1}(\mathcal{A})$, by Burenkov [4, Thm. 3, p. 285] there exists a linear extension operator

$$
E_{\mathcal{N}}: W^{m, p}\left(\Omega_{1}\right) \rightarrow W^{m, p}\left(\mathbb{R}^{N}\right)
$$

with $\left\|E_{\mathcal{N}}\right\|$ depending only on $\mathcal{A}, m$. Let

$$
E_{\mathcal{D}}: W_{0}^{m, p}\left(\Omega_{1}\right) \rightarrow W^{m, p}\left(\mathbb{R}^{N}\right)
$$

be the extension-by-zero operator.

We set

$$
\mathcal{T}_{\mathcal{D}}[u]=\mathcal{T}\left[E_{\mathcal{D}} u\right], \quad \mathcal{T}_{\mathcal{N}}[v]=\mathcal{T}\left[E_{\mathcal{N}} v\right]
$$

for all $u \in W_{0}^{m, p}(\Omega), v \in W^{m, p}(\Omega)$, and

$$
\Omega_{3}=\Omega_{1} \backslash\left(\cup_{j=1}^{s^{\prime}}\left(\Omega_{1} \cap V_{j}\right) \backslash \Omega_{3, j}\right) .
$$

Note that $\Omega_{3} \cap V_{j} \subset \Omega_{3, j}$ for all $j=1, \ldots, s^{\prime}$, hence $\Omega_{3} \subset \Omega_{1} \cap \Omega_{2}$.

By Step 2. it follows that $\mathcal{T}_{\mathcal{N}}$ maps $W^{m, p}\left(\Omega_{1}\right)$ to $W^{m, p}\left(\Omega_{2}\right)$. Moreover, if $u \in W_{0}^{m, p}\left(\Omega_{1}\right)$ then $E_{\mathcal{D}} u$ vanishes outside $\Omega_{1}$, hence $\mathcal{T}_{j}\left[\psi_{j} E_{\mathcal{D}} u\right]$ vanishes outside $\Omega_{2}$ and $\mathcal{T}_{\mathcal{D}}[u] \in W_{0}^{m, p}\left(\Omega_{2}\right)$. Thus $\mathcal{T}_{\mathcal{D}}$ maps $W_{0}^{m, p}\left(\Omega_{1}\right)$ to $W_{0}^{m, p}\left(\Omega_{2}\right)$.

Statement (i) follows by Step 1 and by the properties of the extension operators $E_{\mathcal{N}}, E_{\mathcal{D}}$. The equalities in (3.3) immediately follow by Lemma 3.23. Finally, inequality (3.2) can be deduced by Lemma 3.4 (i) by using exactly the same argument in the proof of [7, Lemma 4.23].

\section{Sharp estimates for the variation of the eigen- values via the Lebesgue measure}

In this section we prove stability estimates for the eigenvalues $\lambda_{n, \mathcal{D}}[\Omega], \lambda_{n, \mathcal{N}}[\Omega]$ defined in Definition 2.8. Recall that $\lambda_{n, \mathcal{D}}[\Omega], \lambda_{n, \mathcal{N}}[\Omega]$ are the eigenvalues of the operators $H_{W_{0}^{m, 2}(\Omega)}, H_{W^{m, 2}(\Omega)}$ respectively.

By $\varphi_{n, \mathcal{D}}[\Omega]$ and $\varphi_{n, \mathcal{N}}[\Omega]$ we denote a sequence of orthonormal eigenfunctions corresponding to $\lambda_{n, \mathcal{D}}[\Omega]$ and $\lambda_{n, \mathcal{N}}[\Omega]$ respectively.

When no distinction between the Dirichlet and the Neumann case is required and we refer to both, we simply write $\lambda_{n}[\Omega], \varphi_{n}[\Omega], H_{\Omega}$ to indicate the eigenvalues and the corresponding eigenfunctions and operators. 
The following statement hold for both Dirichlet and Neumann boundary conditions.

Theorem 4.1 Let $\mathcal{A}=\left(\rho, s, s^{\prime},\left\{V_{j}\right\}_{j=1}^{s},\left\{r_{j}\right\}_{j=1}^{s}\right)$ be an atlas in $\mathbb{R}^{N}, m \in \mathbb{N}$, $M, \theta>0$. For all $\alpha, \beta \in \mathbb{N}_{0}^{N}$ with $|\alpha|=|\beta|=m$, let $A_{\alpha \beta}$ be measurable realvalued functions defined on $\cup_{j=1}^{s} V_{j}$, satisfying $A_{\alpha \beta}=A_{\beta \alpha}$ and condition (2.2).

Let $2<p \leq \infty, 0<M_{n}<\infty$ for all $n \in \mathbb{N}$, and $\mathfrak{A}=\left\{\Omega \in C_{M}^{m-1,1}(\mathcal{A})\right.$ : $\left\|\varphi_{n}[\Omega]\right\|_{W^{m, p}(\Omega)} \leq M_{n}$ for all $\left.n \in \mathbb{N}\right\}$.

Then for each $n \in \mathbb{N}$ there exists $c_{n}>0$ depending only on $n, \mathcal{A}, m, M, \theta, p$, $M_{1}, \ldots, M_{n}$ such that

$$
\lambda_{n}\left[\Omega_{2}\right] \leq \lambda_{n}\left[\Omega_{1}\right]+c_{n}\left|\Omega_{1} \Delta \Omega_{2}\right|^{1-\frac{2}{p}},
$$

for all $\Omega_{1} \in \mathfrak{A}, \Omega_{2} \in C_{M}^{m-1,1}(\mathcal{A})$ such that $\left|\Omega_{1} \triangle \Omega_{2}\right|<c_{n}^{-1}$.

Proof. Let $\Omega_{1} \in \mathfrak{A}$ and $\Omega_{2} \in C_{M}^{m-1,1}(\mathcal{A})$. To shorten our notation we set $\varphi_{n, 1}=\varphi_{n}\left[\Omega_{1}\right]$, for all $n \in \mathbb{N}$. We denote by $\mathcal{L}_{1}$ the space of the finite linear combinations of the eigenfunctions $\varphi_{n, 1}$. Moreover, we define a linear operator

$$
T_{12}: \mathcal{L}_{1} \rightarrow \operatorname{Dom}\left(H_{\Omega_{2}}^{1 / 2}\right)
$$

by setting in the Dirichlet case

$$
T_{12}\left[\varphi_{n, 1}\right]=\mathcal{T}_{\mathcal{D}} \varphi_{n, 1}
$$

and in the Neumann case

$$
T_{12}\left[\varphi_{n, 1}\right]=\mathcal{T}_{\mathcal{N}} \varphi_{n, 1}
$$

for all $n \in \mathbb{N}$. Here

$$
\mathcal{T}_{D}: W_{0}^{m, p}\left(\Omega_{1}\right) \rightarrow W_{0}^{m, p}\left(\Omega_{2}\right) \text { and } \mathcal{T}_{N}: W^{m, p}\left(\Omega_{1}\right) \rightarrow W^{m, p}\left(\Omega_{2}\right)
$$

are the operators provided by Theorem 3.1. Note that $T_{12}$ is well-defined. Indeed, by assumption $\mathcal{L}_{1} \subset W^{m, p}\left(\Omega_{1}\right)$, and in the Dirichlet case $\mathcal{L}_{1} \subset W_{0}^{m, p}\left(\Omega_{1}\right)$. Moreover, $T_{12}$ takes values in $\operatorname{Dom}\left(H_{\Omega_{2}}^{1 / 2}\right)$ because in the Dirichlet case $W_{0}^{m, p}\left(\Omega_{2}\right)$ $\subset W_{0}^{m, 2}\left(\Omega_{2}\right)=\operatorname{Dom}\left(H_{\Omega_{2}}^{1 / 2}\right)$, and in the Neumann case $W^{m, p}\left(\Omega_{2}\right) \subset W^{m, 2}\left(\Omega_{2}\right)=$ $\operatorname{Dom}\left(H_{\Omega_{2}}^{1 / 2}\right)$.

To prove (4.2) we apply the general spectral stability theorem [8, Thm. 3.2]. In the terminology of [8], we need to prove that $T_{12}$ is a 'transition operator' from $H_{\Omega_{1}}$ to $H_{\Omega_{2}}$. To do so, we prove inequalities (4.7) and (4.8) below.

By Theorem 3.1, $T_{12} \varphi_{n}=\varphi_{n}$ on $\Omega_{3}$ where $\Omega_{3}$ is as in Theorem 3.1 (ii). Thus

$$
\begin{aligned}
& \left(H_{\Omega_{2}}^{1 / 2} T_{12} \varphi_{k, 1}, H_{\Omega_{2}}^{1 / 2} T_{12} \varphi_{l, 1}\right)_{L^{2}\left(\Omega_{2}\right)}=Q_{\Omega_{2}}\left(T_{12} \varphi_{k, 1}, T_{12} \varphi_{l, 1}\right) \\
& \quad=Q_{\Omega_{3}}\left(T_{12} \varphi_{k, 1}, T_{12} \varphi_{l, 1}\right)+Q_{\Omega_{2} \backslash \Omega_{3}}\left(T_{12} \varphi_{k, 1}, T_{12} \varphi_{l, 1}\right) \\
& \quad=Q_{\Omega_{3}}\left(\varphi_{k, 1}, \varphi_{l, 1}\right)+Q_{\Omega_{2} \backslash \Omega_{3}}\left(T_{12} \varphi_{k, 1}, T_{12} \varphi_{l, 1}\right) \\
& \quad=Q_{\Omega_{1}}\left(\varphi_{k, 1}, \varphi_{l, 1}\right)-Q_{\Omega_{1} \backslash \Omega_{3}}\left(\varphi_{k, 1}, \varphi_{l, 1}\right)+Q_{\Omega_{2} \backslash \Omega_{3}}\left(T_{12} \varphi_{k, 1}, T_{12} \varphi_{l, 1}\right) \\
& \quad=\left(H_{\Omega_{1}}^{1 / 2} \varphi_{k, 1}, H_{\Omega_{1}}^{1 / 2} \varphi_{l, 1}\right)_{L^{2}\left(\Omega_{1}\right)}-Q_{\Omega_{1} \backslash \Omega_{3}}\left(\varphi_{k, 1}, \varphi_{l, 1}\right)+Q_{\Omega_{2} \backslash \Omega_{3}}\left(T_{12} \varphi_{k, 1}, T_{12} \varphi_{l, 1}\right)
\end{aligned}
$$

for all $k, l \in \mathbb{N}$. By Hölder's inequality

$$
Q_{\Omega_{1} \backslash \Omega_{3}}\left(\varphi_{k, 1}, \varphi_{l, 1}\right) \leq c M_{k} M_{l}\left|\Omega_{1} \backslash \Omega_{3}\right|^{1-\frac{2}{p}}
$$


and by Theorem 3.1 we have

$$
Q_{\Omega_{2} \backslash \Omega_{3}}\left(T_{12} \varphi_{k, 1}, T_{12} \varphi_{l, 1}\right) \leq c M_{k} M_{l}\left|\Omega_{2} \backslash \Omega_{3}\right|^{1-\frac{2}{p}}
$$

and

$$
\left|\Omega_{1} \backslash \Omega_{3}\right|,\left|\Omega_{2} \backslash \Omega_{3}\right| \leq c\left|\Omega_{1} \triangle \Omega_{2}\right|,
$$

where $c>0$ depends only on $\mathcal{A}, m, M, \theta, p$. Thus by (4.3)-(4.6) it follows that

$$
\begin{aligned}
\mid\left(H_{\Omega_{2}}^{1 / 2} T_{12} \varphi_{k, 1},\right. & \left.H_{\Omega_{2}}^{1 / 2} T_{12} \varphi_{l, 1}\right)_{L^{2}\left(\Omega_{2}\right)} \\
& -\left.\left(H_{\Omega_{1}}^{1 / 2} \varphi_{k, 1}, H_{\Omega_{1}}^{1 / 2} \varphi_{l, 1}\right)_{L^{2}\left(\Omega_{1}\right)}\left|\leq \tilde{c}_{5} M_{k} M_{l}\right| \Omega_{1} \Delta \Omega_{2}\right|^{1-\frac{2}{p}}
\end{aligned}
$$

and similarly

$$
\left|\left(T_{12} \varphi_{k, 1}, T_{12} \varphi_{l, 1}\right)_{L^{2}\left(\Omega_{2}\right)}-\left(\varphi_{k, 1}, \varphi_{l, 1}\right)_{L^{2}\left(\Omega_{1}\right)}\right| \leq \tilde{c}_{6} M_{k} M_{l}\left|\Omega_{1} \Delta \Omega_{2}\right|^{1-\frac{2}{p}},
$$

for all $k, l \in \mathbb{N}$, where $\tilde{c}_{5}, \tilde{c}_{6}>0$ depend only on $\mathcal{A}, m, M, \theta, p$.

By (4.7), (4.8) it follows that $T_{12}$ is a transition operator from $H_{\Omega_{1}}$ to $H_{\Omega_{2}}$ with parameters $a_{k l}=\tilde{c}_{5} M_{k} M_{l}, b_{k l}=\tilde{c}_{6} M_{k} M_{l}$ and measure of vicinity $\delta\left(H_{\Omega_{1}}, H_{\Omega_{2}}\right)=$ $\left|\Omega_{1} \triangle \Omega_{2}\right|^{1-\frac{2}{p}}$ (see [8, Def. 3.1]). Thus by [8, Thm. 3.2] it follows that

$$
\lambda_{n}\left[\Omega_{2}\right] \leq \lambda_{n}\left[\Omega_{1}\right]+\left(2 a_{n} \lambda_{n}\left[\Omega_{1}\right]+b_{n}\right) \delta\left(H_{\Omega_{1}}, H_{\Omega_{2}}\right)
$$

if $\delta\left(H_{\Omega_{1}}, H_{\Omega_{2}}\right) \leq\left(2 a_{n}\right)^{-1}$, where $a_{n}=\left(\sum_{k, l=1}^{n} a_{k l}^{2}\right)^{1 / 2}=\tilde{c}_{5} \sum_{k=1}^{n} M_{k}^{2}, b_{n}=$ $\left(\sum_{k, l=1}^{n} b_{k l}^{2}\right)^{1 / 2}=\tilde{c}_{6} \sum_{k=1}^{n} M_{k}^{2}$. Furthermore, by [6, Lemma 3.2] there exists $\Lambda_{n}>0$ depending only on $n, \mathcal{A}, m, \theta$ such that

$$
\lambda_{n}[\Omega] \leq \Lambda_{n}
$$

for all $\Omega \in C_{M}^{m-1,1}(\mathcal{A})$. Thus, inequality (4.2) follows by combining (4.9) and (4.10).

Remark 4.11 It can be traced that starting with (4.3) one can obtain the estimate

$$
\lambda_{n}\left[\Omega_{2}\right] \leq \lambda_{n}\left[\Omega_{1}\right]+c_{n} \sum_{k=1}^{n}\left\|\varphi_{k}\left[\Omega_{1}\right]\right\|_{W^{m, 2}\left(\Omega_{1} \Delta \Omega_{2}\right)}
$$

which in some cases (depending on the properties of $\varphi_{1}\left[\Omega_{1}\right], \ldots, \varphi_{n}\left[\Omega_{1}\right]$ near the boundary of $\Omega_{1}$ ) can be better than estimate (4.2).

It is well-known that if $\Omega_{2} \subset \Omega_{1}$ then $\lambda_{n, \mathcal{D}}\left[\Omega_{1}\right] \leq \lambda_{n, \mathcal{D}}\left[\Omega_{2}\right]$. Thus by Theorem 4.1 we immediately deduce the following corollary concerning Dirichlet eigenvalues (for the proof of the sharpness of estimate (4.13), see Section 5).

Corollary 4.12 Let $\mathcal{A}=\left(\rho, s, s^{\prime},\left\{V_{j}\right\}_{j=1}^{s},\left\{r_{j}\right\}_{j=1}^{s}\right)$ be an atlas in $\mathbb{R}^{N}, m \in \mathbb{N}$, $M, \theta>0$. Let $\Omega_{1} \in C_{M}^{m-1,1}(\mathcal{A})$. For all $\alpha, \beta \in \mathbb{N}_{0}^{N}$ with $|\alpha|=|\beta|=m$, let $A_{\alpha \beta}$ be measurable real-valued functions defined on $\Omega_{1}$, satisfying $A_{\alpha \beta}=A_{\beta \alpha}$ and condition (2.2). 
Assume that $2<p \leq \infty$ and $\varphi_{n, \mathcal{D}}\left[\Omega_{1}\right] \in W^{m, p}\left(\Omega_{1}\right)$ for all $n \in \mathbb{N}$. Then for each $n \in \mathbb{N}$ there exists $c_{n}>0$ depending only on $n, \mathcal{A}, m, M, \theta, p,\left\|\varphi_{k}\left[\Omega_{1}\right]\right\|_{W^{m, p}\left(\Omega_{1}\right)}$ $k=1, \ldots, n$, such that

$$
\lambda_{n, \mathcal{D}}\left[\Omega_{1}\right] \leq \lambda_{n, \mathcal{D}}\left[\Omega_{2}\right] \leq \lambda_{n, \mathcal{D}}\left[\Omega_{1}\right]+c_{n}\left|\Omega_{1} \backslash \Omega_{2}\right|^{1-\frac{2}{p}}
$$

for all $\Omega_{2}$ of class $C_{M}^{m-1,1}(\mathcal{A})$ such that $\Omega_{2} \subset \Omega_{1}$ and $\left|\Omega_{1} \backslash \Omega_{2}\right|<c_{n}^{-1}$.

Moreover, in general the exponent $1-\frac{2}{p}$ in 4.13) cannot be replaced by $1-\frac{2}{p}+\delta$ where $\delta>0$ is a constant independent of $p$.

If we assume that both $\Omega_{1}$ and $\Omega_{2}$ belong to $\mathfrak{A}$ then it is possible to swap $\Omega_{1}$ and $\Omega_{2}$ in (4.2). In this way we obtain a two-sided estimate for both Dirichlet and Neumann eigenvalues without assuming that $\Omega_{2} \subset \Omega_{1}$ as in Corollary 4.12,

Corollary 4.14 Let $\mathcal{A}=\left(\rho, s, s^{\prime},\left\{V_{j}\right\}_{j=1}^{s},\left\{r_{j}\right\}_{j=1}^{s}\right)$ be an atlas in $\mathbb{R}^{N}, m \in \mathbb{N}$, $M, \theta>0$. For all $\alpha, \beta \in \mathbb{N}_{0}^{N}$ with $|\alpha|=|\beta|=m$, let $A_{\alpha \beta}$ be measurable real-valued functions defined on $\cup_{j=1}^{s} V_{j}$, satisfying $A_{\alpha \beta}=A_{\beta \alpha}$ and condition (2.2).

Let $2<p \leq \infty$ and let $\mathfrak{A}$ be a family of open sets of class $C_{M}^{m-1,1}(\mathcal{A})$ such that for each $n \in \mathbb{N}$ condition (1.2) is satisfied.

Then for each $n \in \mathbb{N}$ there exists $c_{n}>0$ depending only on $n, \mathcal{A}, m, M, \theta, p$, $\sup _{\Omega \in \mathfrak{A}}\left\|\varphi_{k}[\Omega]\right\|_{W^{m, p}(\Omega)} k=1, \ldots, n$, such that

$$
\left|\lambda_{n}\left[\Omega_{1}\right]-\lambda_{n}\left[\Omega_{2}\right]\right| \leq c_{n}\left|\Omega_{1} \Delta \Omega_{2}\right|^{1-\frac{2}{p}},
$$

for all $\Omega_{1}, \Omega_{2} \in \mathfrak{A}$ such that $\left|\Omega_{1} \triangle \Omega_{2}\right|<c_{n}^{-1}$.

If $\mathfrak{A}$ is a family of open sets with sufficiently smooth boundaries then condition (1.2) is satisfied with $p=\infty$.

Lemma 4.16 Let $\mathcal{A}=\left(\rho, s, s^{\prime},\left\{V_{j}\right\}_{j=1}^{s},\left\{r_{j}\right\}_{j=1}^{s}\right)$ be an atlas in $\mathbb{R}^{N}, m \in \mathbb{N}$, $B, M, \theta>0$. For all $\alpha, \beta \in \mathbb{N}_{0}^{N}$ with $|\alpha|=|\beta|=m$, let $A_{\alpha \beta} \in C^{m}\left(\overline{\cup_{j=1}^{s} V_{j}}\right)$ satisfy $A_{\alpha \beta}=A_{\beta \alpha},\left\|A_{\alpha \beta}\right\|_{c^{m}\left(\overline{\mathrm{U}_{j=1}^{s} V_{j}}\right)} \leq B$, and condition (2.2). Then $\varphi_{n}[\Omega] \in$ $W^{2 m-1, \infty}(\Omega)$ and there exists $C>0$ depending only on $\mathcal{A}, m, B, M, \theta$ such that

$$
\left\|\varphi_{n}[\Omega]\right\|_{W^{k, \infty}(\Omega)} \leq C\left(1+\lambda_{n}[\Omega]\right)^{\frac{N}{4 m}+\frac{k}{2 m}}
$$

for all $k=0, \ldots, 2 m-1$ and $\Omega \in C_{M}^{2 m}(\mathcal{A})$.

Proof. It is well-known that under our regularity assumptions $\operatorname{Dom}(H) \subset$ $W^{2 m, 2}(\Omega)$ (see e.g., Agmon [2, Sec. 9]). Moreover, since the coefficients $A_{\alpha \beta}$ are of class $C^{m}$ and we impose either Dirichlet or Neumann boundary conditions, we can resort to the general setting of Agmon [1] (see [2, pp. 141-143] for details).

Thus, by [1, Thm. 1.1 and the Lemma on p.131] it follows that if $u \in \operatorname{Dom}(H)$ and $H u \in L^{p}(\Omega)$ for some $p>1$ then $u \in W^{2 m, p}(\Omega)$ and

$$
\|u\|_{W^{2 m, p}(\Omega)} \leq c\left(\|H u\|_{L^{p}(\Omega)}+\|u\|_{L^{p}(\Omega)}\right),
$$

where $c$ is a positive constant. In particular if $\varphi$ is an eigenfunction corresponding to an eigenvalue $\lambda$ and $\varphi \in L^{p}(\Omega)$ then

$$
\|u\|_{W^{2 m, p}(\Omega)} \leq c(1+\lambda)\|u\|_{L^{p}(\Omega)} .
$$


By the apriori estimate (4.19) and a bootstrap argument one can finally prove estimate (4.17) (see for instance Burenkov and Lamberti [8, Thm. 5.1] where in the proof one has simply to replace [8, (5.5)] by (4.19)).

By Corollary 4.14 and Lemma 4.16 we immediately deduce the validity of the following

Corollary 4.20 Let $\mathcal{A}=\left(\rho, s, s^{\prime},\left\{V_{j}\right\}_{j=1}^{s},\left\{r_{j}\right\}_{j=1}^{s}\right)$ be an atlas in $\mathbb{R}^{N}, m \in \mathbb{N}$, $B, M, \theta>0$. For all $\alpha, \beta \in \mathbb{N}_{0}^{N}$ with $|\alpha|=|\beta|=m$, let $A_{\alpha \beta} \in C^{m}\left(\overline{\bigcup_{j=1}^{s} V_{j}}\right)$ satisfy $A_{\alpha \beta}=A_{\beta \alpha},\left\|A_{\alpha \beta}\right\|_{c^{m}\left(\overline{\cup_{j=1}^{s} V_{j}}\right)} \leq B$, and condition (2.2). Then for all $n \in \mathbb{N}$ there exists $c_{n}>0$ depending only on $n, \mathcal{A}, m, B, M, \theta$ such that

$$
\left|\lambda_{n}\left[\Omega_{1}\right]-\lambda_{n}\left[\Omega_{2}\right]\right| \leq c_{n}\left|\Omega_{1} \triangle \Omega_{2}\right|,
$$

for all $\Omega_{1}, \Omega_{2} \in C_{M}^{2 m}(\mathcal{A})$ satisfying $\left|\Omega_{1} \triangle \Omega_{2}\right|<c_{n}^{-1}$.

\section{An example}

We consider an example which proves that in the class of Lipschitz domains the exponent in estimates (1.3) and (1.4) cannot, in general, be larger than 1 $2 / p$. For this purpose we consider the Dirichlet and Neumann Laplacians on the circular sector $\Omega \subset \mathbb{R}^{2}$ of radius $R=1$ and angle $2 \beta$ with $0<\beta<\pi$. In polar coordinates

$$
\Omega=\{(\rho, \theta): 0<\rho<1,-\beta<\theta<\beta\} .
$$

For $0<\epsilon<1$ we consider the deformation $\Omega(\epsilon)$ of $\Omega$ given by

$$
\Omega(\epsilon)=\{(\rho, \theta): \epsilon<\rho<1,-\beta<\theta<\beta\} .
$$

Here we are interested in the behavior of the eigenvalues of the Dirichlet and Neumann Laplacians on $\Omega(\epsilon)$ as $\epsilon \rightarrow 0$.

In the case of the Dirichlet Laplacian on $\Omega$ all the eigenvalues are the positive solutions of the equations

$$
J_{\nu}(\sqrt{\lambda})=0,
$$

where $J_{\nu}$ is the Bessel function of the first kind and order $\nu$, with $\nu=\pi k /(2 \beta)$, $k \in \mathbb{N}$.

Note that $\nu>1 / 2$ for all $0<\beta<\pi, k \in \mathbb{N}$, and that $\nu<1$ if an only if $k=1$ and $\pi / 2<\beta<\pi$.

For our purposes, it is enough to restrict our attention to the case $\nu \notin \mathbb{N}$ : in this case the eigenvalues of the Dirichlet Laplacian on $\Omega(\epsilon)$ are the positive solutions of the cross-product equations

$$
\left.J_{\nu}(\sqrt{\lambda}) J_{-\nu}(\epsilon \sqrt{\lambda})\right)-J_{-\nu}(\sqrt{\lambda}) J_{\nu}(\epsilon \sqrt{\lambda})=0 .
$$

Recall that for a Bessel function of the first kind and order $\mu(\mu \neq-1,-2, \ldots)$ we have $J_{\mu}(s)=s^{\mu} H_{\mu}\left(s^{2}\right), s \in \mathbb{R}$, where $H_{\mu}$ is an analytic function such that $H_{\mu}(0) \neq 0$, see $([12, \S 9.1 .10])$.

Assume that $\lambda_{*}$ is a fixed eigenvalue of the Dirichlet Laplacian on $\Omega$, i.e., $\lambda_{*}$ is a fixed zero of $H_{\nu}$. It is known that $H_{-\nu}\left(\lambda_{*}\right) \neq 0$. Thus, in a sufficiently small 
small neighborhood of $\lambda_{*}$ and for sufficiently small $\epsilon \geq 0$, equation (5.4) can be rewritten as

$$
f(\lambda)-\epsilon^{2 \nu} f\left(\epsilon^{2} \lambda\right)=0,
$$

where $f(\lambda)=H_{\nu}(\lambda) / H_{-\nu}(\lambda)$ is an analytic function in a neighborhood of zero and in a neighborhood of $\lambda_{*}$.

It is immediate to verify that if $\epsilon=0$ then the positive solutions of (5.3) coincide with the positive solutions of equation (5.5). Thus, for each $0 \leq \epsilon<1$ the eigenvalues $\lambda$ of the Dirichlet Laplacian on $\Omega(\epsilon)$ are exactly the zeros of equation (5.5) (here it is understood that $\Omega(0)=\Omega$ ).

We set $\delta=\epsilon^{2 \nu}$, so that equation (5.5) can be rewritten as

$$
f(\lambda)-\delta f\left(\delta^{\frac{1}{\nu}} \lambda\right)=0 .
$$

Observe that the left-hand side of equation (5.6) defines a function of class $C^{1}$ in the variables $\delta, \lambda$, for all $(\delta, \lambda)$ a neighborhood of the point $\left(0, \lambda_{*}\right)$. Note that $H_{\nu}^{\prime}\left(\lambda_{*}\right) \neq 0$ since all positive zeros of the Bessel functions $J_{\nu}$ are simple, see [12, 9.5.2]. Thus, $f^{\prime}\left(\lambda_{*}\right) \neq 0$ and and by the Implicit Function Theorem the zeros of equation (5.6) in a neighborhood of $\left(0, \lambda_{*}\right)$ are given by the graph of a function $\delta \mapsto \lambda(\delta)$ of class $C^{1}$ such that $\lambda(0)=\lambda_{*}$. Moreover, since the derivative of $f(\lambda)-\delta f\left(\delta^{\frac{1}{\nu}} \lambda\right)$ with respect to $\delta$ at the point $\left(0, \lambda_{*}\right)$ is equal to $-f(0)$ then by the Implicit Function Theorem we have that

$$
\lambda(\delta)=\lambda_{*}+\frac{f(0)}{f^{\prime}\left(\lambda_{*}\right)} \delta+o(\delta), \quad \text { as } \delta \rightarrow 0^{+} .
$$

Note that $f(0) \neq 0$. This clearly implies that

$$
\left|\lambda(\epsilon)-\lambda_{*}\right|=C|\Omega \backslash \Omega(\epsilon)|^{\nu}+o\left(|\Omega \backslash \Omega(\epsilon)|^{\nu}\right), \quad \text { as } \epsilon \rightarrow 0^{+},
$$

where $C$ is a positive constant.

We note that the eigenspace of the Dirichlet Laplacian on $\Omega$ corresponding to the eigenvalue $\lambda_{*}$ is spanned by the function $U$ defined in polar coordinates by

$$
U(\rho, \theta)=J_{\nu}\left(\rho \sqrt{\lambda_{*}}\right) \sin \nu(\theta+\beta)=\left(\rho \sqrt{\lambda_{*}}\right)^{\nu} H_{\nu}\left(\rho^{2} \lambda_{*}\right) \sin \nu(\theta+\beta),
$$

for all $0<\rho<1,-\beta<\theta<\beta$. Clearly, $U \in L^{\infty}(\Omega)$ as expected, and if $\nu \geq 1$ then $\nabla U \in L^{\infty}(\Omega)$, whilst if $1 / 2<\nu<1$ then $\nabla U \in L^{p}(\Omega)$ if and only if $1 \leq p<2 /(1-\nu)$. Thus by applying estimate (1.4) we obtain that if $1 / 2<\nu<1$ then for any $0<\gamma<\nu$ there exists $c_{\gamma}>0$ such that

$$
\left|\lambda(\epsilon)-\lambda_{*}\right| \leq c_{\gamma}|\Omega \backslash \Omega(\epsilon)|^{\gamma},
$$

if $|\Omega \backslash \Omega(\epsilon)|<c_{\gamma}^{-1}$, whilst if $\nu \geq 1$ then there exists $c>0$ such that

$$
\left|\lambda(\epsilon)-\lambda_{*}\right| \leq c|\Omega \backslash \Omega(\epsilon)|,
$$

if $|\Omega \backslash \Omega(\epsilon)|<c^{-1}$.

In the case of the Neumann Laplacian, equations (5.3), (5.4) should be replaced by equations

$$
J_{\nu}^{\prime}(\sqrt{\lambda})=0
$$

and 


$$
\left.J_{\nu}^{\prime}(\sqrt{\lambda}) J_{-\nu}^{\prime}(\epsilon \sqrt{\lambda})\right)-J_{-\nu}^{\prime}(\sqrt{\lambda}) J_{\nu}^{\prime}(\epsilon \sqrt{\lambda})=0,
$$

respectively. By writing $J_{\mu}^{\prime}(s)=s^{\mu-1} K_{\mu}\left(s^{2}\right)$ where $K_{\mu}$ is a suitable analytic function not vanishing at zero, one can easily see that in the case of the Neumann Laplacian in equation (5.5) one should simply replace the function $f$ by the function $g(\lambda)=K_{\nu}(\lambda) / K_{-\nu}(\lambda)$. Note that $K_{\nu}^{\prime}\left(\lambda_{*}\right) \neq 0$ since all positive zeros of $J_{\nu}^{\prime}$ are simple (use directly the Bessel equation of order $\nu$ and Watson [13, §15.3, (3), p. 486]). Thus, one can apply the same argument used above and prove that (5.8) holds also for the eigenvalues of the Neumann Laplacian.

Note that the eigenspace of the Neumann Laplacian on $\Omega$ corresponding to a positive eigenvalue $\lambda_{*}$ is spanned by the function $V$ defined in polar coordinates by

$$
V(\rho, \theta)=J_{\nu}\left(\rho \sqrt{\lambda_{*}}\right) \cos \nu(\theta+\beta)=\left(\rho \sqrt{\lambda_{*}}\right)^{\nu} H_{\nu}\left(\rho^{2} \lambda_{*}\right) \cos \nu(\theta+\beta),
$$

for all $0<\rho<1,-\beta<\theta<\beta$. Thus also for the Neumann Laplacian, we conclude that inequality (1.3) implies (5.10) and (5.11).

Clearly, in both the cases of Dirichlet and Neumann boundary conditions, (5.8) shows that if $k=1$ and $\pi / 2<\beta<\pi(\Longleftrightarrow 1 / 2<\nu<1)$ then the exponent $\gamma$ in (5.10) cannot be larger than $\nu$. Thus, in general, the exponent in the right hand-side of estimates (1.3), (1.4) in the class of Lipschitz domains cannot be larger than $1-2 / p$. However, (5.8) and (5.11) also show that for special domains and special values of the indices $n$ one may find better exponents in the right hand-side of estimates (1.3), (1.4).

Note that in this example the domains $\Omega$ and $\Omega(\epsilon)$ are of class $C^{0,1}$ but not of class $C_{M}^{0,1}(\mathcal{A})$ for fixed atlas $\mathcal{A}$ and $M>0$. In the proof below the domains $\Omega(\epsilon)$ will be modified in an appropriate way in order to define suitable domains $\tilde{\Omega}(\epsilon)$ belonging to the same class $C_{M}^{0,1}(\mathcal{A})$.

Proof of the sharpness of the exponent $1-2 / p$ in (4.13) for $N=2$, $m=1, n=1$. In this proof, by $\lambda_{1, \mathcal{D}}[\mathcal{U}]$ we denote the first eigenvalue of the Dirichlet Laplacian defined on a bounded domain $\mathcal{U}$ in $\mathbb{R}^{2}$.

Let $\Omega$ be the domain defined by (5.1) with $\pi / 2<\beta<\pi$. For all $\epsilon \in] 0,1[$ we set

$$
\tilde{\Omega}(\epsilon)=\left\{x=\left(x_{1}, x_{2}\right) \in \mathbb{R}^{2}: g\left(x_{2}\right)<x_{1}, \quad|x|<1\right\},
$$

where $g\left(x_{2}\right)=\epsilon-\left|x_{2}\right| \tan \frac{\beta}{2}$ if $\left|x_{2}\right| \leq \epsilon \sin \beta$, and $g\left(x_{2}\right)=\left|x_{2}\right| \cot \beta$ if $\left|x_{2}\right|>\epsilon \sin \beta$.

It is easy to see that

$$
\Omega(\epsilon) \subset \tilde{\Omega}(\epsilon) \subset \Omega(A \epsilon) \subset \Omega,
$$

for all $\epsilon \in] 0,1\left[\right.$, where $\Omega(\epsilon)$ is defined by (5.2) and $A=\cos \frac{\beta}{2}$. By monotonicity it follows that

$$
\lambda_{1, \mathcal{D}}[\Omega] \leq \lambda_{1, \mathcal{D}}[\Omega(A \epsilon)] \leq \lambda_{1, \mathcal{D}}[\tilde{\Omega}(\epsilon)] \leq \lambda_{1, \mathcal{D}}[\Omega(\epsilon)] .
$$

Since the eigenfunctions corresponding to the first eigenvalue of the Dirichlet Laplacian are the only eigenfunctions which do not change sign, it follows that the eigenspace corresponding to the eigenvalue $\lambda_{1, \mathcal{D}}[\Omega]$ is spanned by (5.9) with $\nu=\pi /(2 \beta)$. Thus, the asymptotic behavior of $\lambda_{1, \mathcal{D}}[\Omega(\epsilon)]$ is given by (5.8) with $\nu=\pi /(2 \beta)$, hence

$$
\lambda_{1, \mathcal{D}}[\Omega(\epsilon)]=\lambda_{1, \mathcal{D}}[\Omega]+C|\Omega \backslash \Omega(\epsilon)|^{\frac{\pi}{2 \beta}}+o\left(|\Omega \backslash \Omega(\epsilon)|^{\frac{\pi}{2 \beta}}\right), \quad \text { as } \epsilon \rightarrow 0^{+} .
$$


By combining (5.15) and (5.16) it follows that

$$
C_{1}|\Omega \backslash \tilde{\Omega}(\epsilon)|^{\frac{\pi}{2 \beta}} \leq\left|\lambda_{1, \mathcal{D}}[\tilde{\Omega}(\epsilon)]-\lambda_{1, \mathcal{D}}[\Omega]\right| \leq C_{2}|\Omega \backslash \tilde{\Omega}(\epsilon)|^{\frac{\pi}{2 \beta}},
$$

for all sufficiently small $\epsilon$, where $C_{1}, C_{2}$ are positive constants independent of $\epsilon$.

We now apply Corollary 4.12 to the Dirichlet Laplacian with $\Omega_{1}=\Omega$ and $\Omega_{2}=\tilde{\Omega}(\epsilon)$. It is clear that there exists an atlas $\mathcal{A}$ and $M>0$ such that $\Omega$ and $\tilde{\Omega}(\epsilon)$ are of class $C_{M}^{0,1}(\mathcal{A})$ for all $\left.\epsilon \in\right] 0,1 / 2$. Moreover, by formula (5.9) it follows that if $1 \leq p<4 \beta /(2 \beta-\pi)$ then the eigenfunctions $\varphi_{n, \mathcal{D}}\left[\Omega_{1}\right]$ of the Dirichlet Laplacian in $\Omega_{1}$ belong to $W^{1, p}\left(\Omega_{1}\right)$ for all $n \in \mathbb{N}$. Thus the assumptions of Corollary 4.12 are satisfied for such range of $p$. Assume now by contradiction that under the assumption of Corollary 4.12 estimate (4.13) is valid with $\left|\Omega_{1} \backslash \Omega_{2}\right|^{1-2 / p+\delta}$ replacing $\left|\Omega_{1} \backslash \Omega_{2}\right|^{1-2 / p}$, where $\delta$ is a positive constant independent of $p$. Since $\lim _{p \rightarrow 4 \beta /(2 \beta-\pi)} 1-2 / p=\pi /(2 \beta)$, by choosing $p$ sufficiently close to $4 \beta /(2 \beta-\pi)$ it follows that the second inequality in (5.17) holds with $\mid \Omega \backslash \tilde{\Omega}(\epsilon)^{\frac{\pi}{2 \beta}+\mu}$ replacing $|\Omega \backslash \tilde{\Omega}(\epsilon)|^{\frac{\pi}{2 \beta}}$ for some $\mu>0$ and this contradicts the first inequality in (5.17) as $\epsilon \rightarrow 0^{+}$.

Acknowledgments: The authors are thankful to Professor M. Marletta who carried out some numerical calculations confirming the sharpness of the exponent $1-2 / p$ in estimates (4.2), (4.13), (4.15) and thus encouraged us to give analytic proof of the sharpness of this exponent.

We also note that further numerical calculations and some analytic computations in the spirit of Section 5 were carried out by the student N.A. Oliver under the supervision of Professor M. Marletta.

This research was supported by the research project "Problemi di stabilità per operatori differenziali" of the University of Padova, Italy and by the research project PRIN 2008 "Aspetti geometrici delle equazioni alle derivate parziali e questioni connesse". The first author was also supported by the grant of RFBR Russian Foundation for Basic Research (projects 09-01-00093-A, 11-01-00744-A).

The authors are also especially thankful to the anonymous Referee for critical analysis of the manuscript and valuable comments which helped to improve the presentation of the results.

\section{References}

[1] S. Agmon, On the eigenfunctions and on the eigenvalues of general elliptic boundary value problems, Comm. Pure Appl. Math., 15, 119-147, 1962.

[2] S. Agmon, Lectures on elliptic boundary value problems, D. Van Nostrand Co., Inc., Princeton, N.J.-Toronto-London, 1965.

[3] G. Barbatis, V. Burenkov and P.D. Lamberti, Stability estimates for resolvents, eigenvalues and eigenfunctions of elliptic operators on variable domains, in: Laptev, Ari (ed.), Around the research of Vladimir Maz'ya. II. Partial differential equations. Dordrecht: Springer; Novosibirsk: Tamara Rozhkovskaya Publisher. International Mathematical Series (New York), 12, 23-60, 2010. 
[4] V.I. Burenkov, Sobolev spaces on domains, B.G. Teubner, Stuttgart-Leipzig, 1998.

[5] V.I. Burenkov and E.B. Davies, Spectral stability of the Neumann Laplacian, J. Differential Equations, 186, 485-508, 2002.

[6] V.I. Burenkov and P.D. Lamberti, Spectral stability of higher order uniformly elliptic operators, in Sobolev Spaces in Mathematics II. Applications in Analysis and Partial Differential Equations (to the centenary of Sergey Sobolev), edited by V. Maz'ya, International Mathematical Series, Vol. 9, Springer, New York, 2009.

[7] V.I. Burenkov and P.D. Lamberti, Spectral stability of Dirchlet second order uniformly elliptic operators , J. Differential Equations, 244, 1712-1740, 2008.

[8] V.I. Burenkov and P.D. Lamberti, Spectral stability of general non-negative self-adjoint operators with applications to Neumann-type operators, J. Differential Equations, 233, 345-379, 2007.

[9] V.I. Burenkov, P.D. Lamberti and M. Lanza de Cristoforis, Spectral stability of nonnegative selfadjoint operators, Sovrem. Mat. Fundam. Napravl., 15, 76-111, 2006 (in Russian. English transl. in J. Math. Sci. (N.Y.), 149, 1417$1452,2008)$.

[10] J. Nečas, Les méthodes directes en théorie des équations elliptiques, Masson et Cie., Paris, 1967.

[11] V.G. Prikazhchikov and A.A. Klunnik, Estimates for eigenvalues of a biharmonic operator perturbed by the variation of a domain, J. Math. Sci. (New York), 84, pp. 1298-1303, 1997.

[12] F.W.J. Olver, Bessel functions of integer order, in Handbook of mathematical functions, Edited by M. Abramowitz and I.A. Stegun, Dover Publications, Inc., New York, 1965, p. 355-433.

[13] G.N. Watson, A treatise on the theory of Bessel Functions, Cambridge University Press, Cambridge, 1966.

V.I. Burenkov, Faculty of Mechanics and Mathematics, L.N. Gumlyov Eurasian National University, 5 Munaitpasov Str., 010008 Astana, Kazakhstan.

P.D. Lamberti, Dipartimento di Matematica Pura ed Applicata, Università degli Studi di Padova, Via Trieste 63, 35121 Padova, Italy. 